\title{
Endothelial Nogo-B regulates sphingolipid biosynthesis to promote pathological cardiac hypertrophy during chronic pressure overload
}

\author{
Yi Zhang, ${ }^{1}$ Yan Huang, ${ }^{2}$ Anna Cantalupo, ${ }^{1}$ Paula S. Azevedo, ${ }^{3}$ Mauro Siragusa, ${ }^{4}$ Jacek Bielawski, ${ }^{5}$ \\ Frank J. Giordano, ${ }^{2}$ and Annarita Di Lorenzo ${ }^{1}$ \\ 'Center for Vascular Biology, Department of Pathology and Laboratory Medicine, Weill Cornell Medicine, \\ Cornell University, New York, New York, USA. ${ }^{2}$ Section of Cardiovascular Medicine, Department of Internal Medicine, \\ and Vascular Biology and Therapeutic Program, Yale University School of Medicine, New Haven, Connecticut, USA. \\ ${ }^{3}$ Department of Internal Medicine, Botucatu Medical School, University of Estadual Paulista, Botucatu, São Paulo, \\ Brazil. ${ }^{4}$ Center for Molecular Medicine, Institute for Vascular Signalling, Goethe University Frankfurt, Frankfurt, Germany \\ ${ }^{5}$ Lipidomics Mass Spectrometry Facility, Department of Biochemistry and Molecular Biology, \\ Medical University of South Carolina, Charleston, South Carolina, USA.
}

We recently discovered that endothelial Nogo-B, a membrane protein of the ER, regulates vascular function by inhibiting the rate-limiting enzyme, serine palmitoyltransferase (SPT), in de novo sphingolipid biosynthesis. Here, we show that endothelium-derived sphingolipids, particularly sphingosine-1-phosphate (S1P), protect the heart from inflammation, fibrosis, and dysfunction following pressure overload and that Nogo-B regulates this paracrine process. SPT activity is upregulated in banded hearts in vivo as well as in TNF- $\alpha$-activated endothelium in vitro, and loss of Nogo removes the brake on SPT, increasing local S1P production. Hence, mice lacking Nogo-B, systemically or specifically in the endothelium, are resistant to the onset of pathological cardiac hypertrophy. Furthermore, pharmacological inhibition of SPT with myriocin restores permeability, inflammation, and heart dysfunction in Nogo-A/B-deficient mice to WT levels, whereas SEW2871, an S1P, receptor agonist, prevents myocardial permeability, inflammation, and dysfunction in WT banded mice. Our study identifies a critical role of endothelial sphingolipid biosynthesis and its regulation by Nogo-B in the development of pathological cardiac hypertrophy and proposes a potential therapeutic target for the attenuation or reversal of this clinical condition.

Conflict of interest: The authors have declared that no conflict of interest exists.

Submitted: November 18, 2015 Accepted: March 18, 2016 Published: April 21, 2016

Reference information: JCI Insight. 2016;1(5):e85484. doi:10.1172/jci.insight.85484.

\section{Introduction}

Cardiac hypertrophy induced by pressure overload is associated with pathological myocardial and vascular changes that can lead to cardiac dysfunction and, ultimately, heart failure. Although several key molecular pathways regulating cardiac hypertrophy have been defined $(1,2)$, not all hypertrophy is detrimental, and a clear understanding of the mechanisms that differentially control adaptive versus pathological hypertrophy remains elusive. This knowledge gap is underscored by the fact that, despite current therapies, mortality among patients with heart failure remains high, suggesting the need for alternative targets and therapeutic options for preservation of cardiac function.

Studies to elucidate the mechanisms of pathological cardiac hypertrophy have largely focused on cardiomyocytes, whereas the contribution of the vasculature, including paracrine roles, remains poorly defined. In pressure-overloaded hearts, tension is distributed throughout the whole coronary artery tree, and the vascular wall becomes a primary source of proinflammatory signals leading to myocardial inflammation and damage (3-6). Endothelial dysfunction is recognized as an early event in the ontogeny of heart failure and contributes to the onset of myocardial inflammation, leading to fibrosis, impaired coronary perfusion, and heart dysfunction. Thus, clarification of the mechanisms underlying the contribution of the vasculature to pathological cardiac hypertrophy is of great significance. 
Recent studies suggest an important role for sphingolipids and sphingosine-1-phosphate (S1P) receptors (S1PR1-3) in cardiovascular development and homeostasis $(7,8)$. Whereas S1P plays an active role in heart development (9), evidence suggests a cardioprotective function of S1P during ischemia in adults $(10,11)$. In the vasculature, S1P exerts important effects on the endothelium, including barrier enhancement (12), which is primarily dependent on S1PR1 activation and cortical actin reorganization (13), and activation of endothelial nitric oxide synthase (eNOS) through S1PR1 and S1PR3. Thus, many of the beneficial effects of S1P are mediated by NO, including regulation of vessel tone and cardioprotective activity $(14,15)$.

We recently identified endothelial-derived S1P as a critical regulator of blood flow and pressure through autocrine effects on S1PR1 and discovered a novel mechanism of regulation of de novo synthesis of endothelial sphingolipid by Nogo-B, a membrane protein of the endoplasmic reticulum that is expressed at a high level in blood vessels (16). Nogo-B modulates production of local sphingolipids, particularly S1P, to affect systemic vascular function, inflammation (17), and hypertension (18). Mechanistically, Nogo-B binds to and inhibits serine palmitoyltransferase (SPT), the rate-limiting enzyme of the sphingolipid de novo synthesis pathway controlling local endothelial S1P production and its autocrine G protein-coupled receptor-dependent signaling actions (18).

Here, we show that Nogo-B-regulated sphingolipids and S1P within the myocardial endothelium have important cardioprotective functions in a mouse model of heart failure. Mice lacking Nogo-A/B systemically and specifically in endothelial cells are protected from permeability, inflammation, and fibrosis following chronic pressure overload and develop physiological cardiac hypertrophy with preservation of heart function.

The role of local sphingolipids in regulating endothelial cell function to affect heart function suggests that dysregulation of sphingolipid metabolism and signaling in the endothelium may have a causative role in the pathogenesis of heart failure.

\section{Results}

Nogo- $A$ and Nogo-B isoform expression in pressure-overloaded hearts. Previous studies showed the upregulation expression of the reticulon 4 (Rtn4) gene, which encodes Nogo, in pressure-overloaded hearts (19), with Nogo-A expression increased in the hearts of patients affected by dilated cardiomyopathy (20) and by congenital heart defects (21). These data suggest that Nogo proteins might play an important role in the pathogenesis of heart failure, in addition to being markers of cardiomyopathy. First, we assessed the expression pattern of both Nogo-A and -B in sections of murine and human hearts. To this end, we performed immunofluorescence staining of Nogo using a polyclonal antibody that recognizes both Nogo-A and Nogo-B. In both mouse and human hearts (Figure 1A), Nogo-A/B was expressed in the endothelial and smooth muscle cells of the coronary vasculature, capillaries, and fibroblasts but not in cardiomyocytes (Supplemental Figure 1A, control; supplemental material available online with this article; doi:10.1172/jci.insight.85484DS1). Next, to evaluate the pattern of Nogo-A/B expression in pathological hearts, we subjected mice to transverse aortic constriction (TAC) (22). Western blot analysis of the hearts showed that Nogo-A and Nogo-B were upregulated at 3 days and 2 weeks after TAC versus sham-operated mice and were almost undetectable 3 months after TAC (Figure 1B).

These findings were supported by immunofluorescence staining of heart sections showing that NogoA/B expression was transiently increased in cardiomyocytes at 3 days after TAC (the percentage of NogoA/B-positive cardiomyocytes was $21.36 \% \pm 4.67 \%$ vs. $1.09 \% \pm 0.20 \%$ in sham mice) and markedly downregulated in the hearts at 3 months after TAC (Figure 1C). Interestingly, endothelial cells, but not smooth muscle cells, of the coronary arteries showed marked upregulation of Nogo-A/B at 3 days and 2 weeks after TAC (Supplemental Figure 1A and Supplemental Figure 2). To dissect the expression of Nogo-A versus Nogo-B in cardiomyocytes and vasculature of control and hypertrophic hearts, we stained serial heart sections with an antibody binding specifically to Nogo-A and an antibody recognizing both Nogo-A and Nogo-B. In control mice, Nogo-A was undetectable, suggesting that under physiological conditions only Nogo-B is expressed in the vasculature of the heart (Figure 1D). Interestingly, pressure overload induced the expression of Nogo-A specifically and exclusively in cardiomyocytes, although expression was transient, with a maximum of approximately $20 \%$ Nogo-A-positive cardiomyocytes at 3 days after TAC (Figure 1, C and D), whereas Nogo-B was expressed in the vasculature, including endothelial cells, vascular smooth muscle cells, and fibroblasts. Western blot analysis further indicated that Nogo-A, but not Nogo-B, was expressed in cardiomyocytes at 3 days after TAC (Figure 1E). Taken together, these data suggest that chronic pressure overload of the heart induces transient and specific upregulation of Nogo-B in the endothelial cells of the coronary vasculature and of Nogo-A in cardiomyocytes. 
A

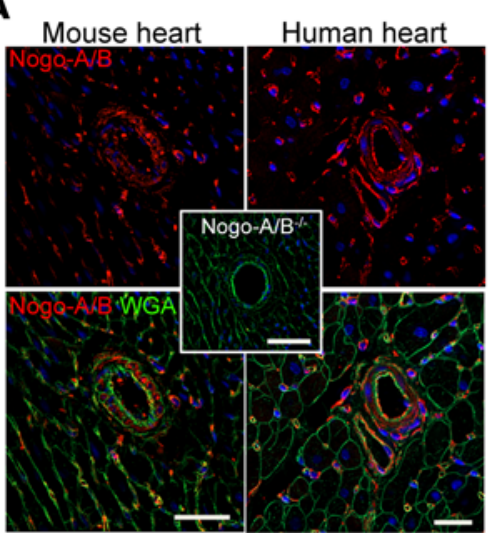

B

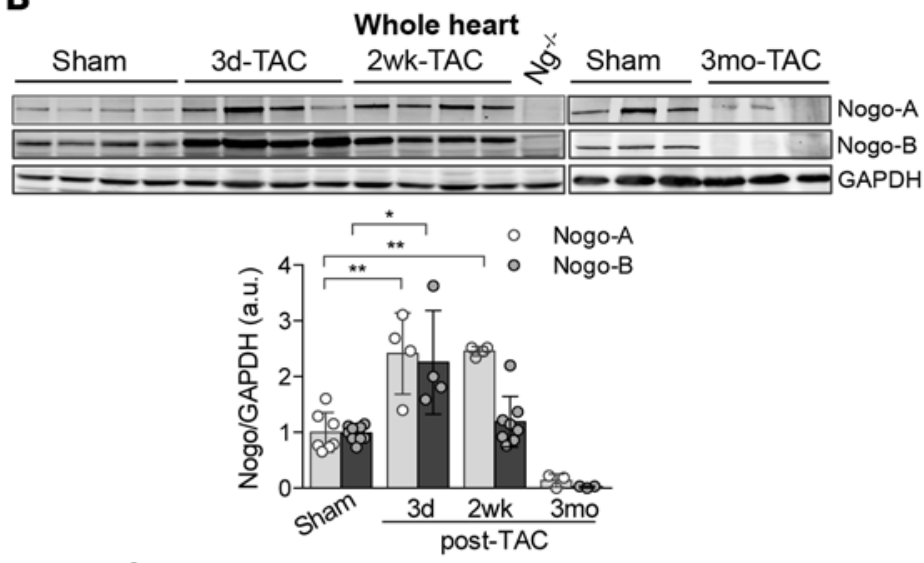

C

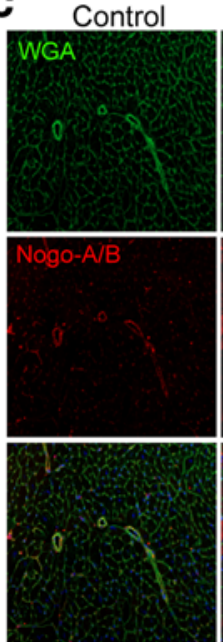

$\mathrm{Ng}^{+} \mathrm{CM} 1.09 \pm 0.20$

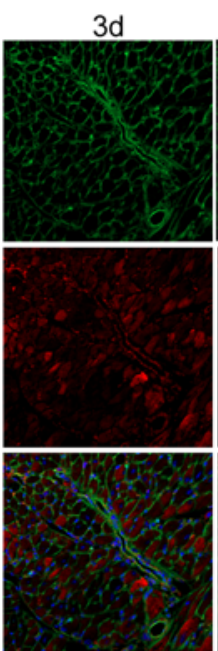

$21.36 \pm 4.67^{\star \star \star}$

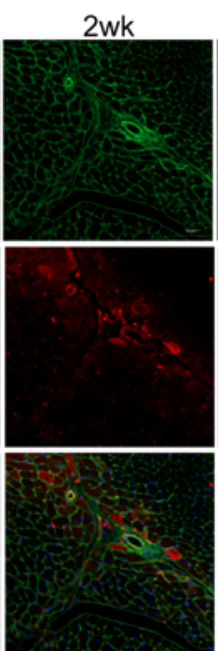

$11.54 \pm 2.11$

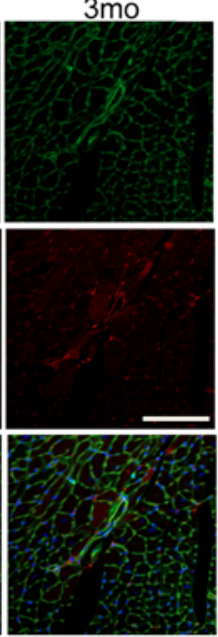

$4.96 \pm 2.11$
D
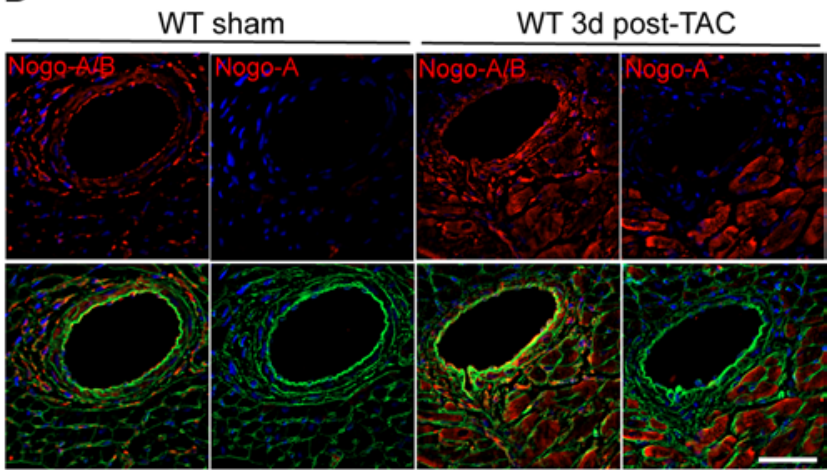

E

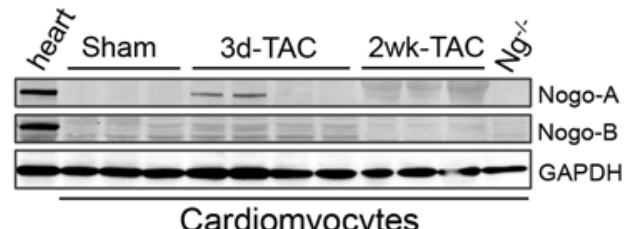

Figure 1. Transient and cell-type-specific overexpression of Nogo-A and Nogo-B in banded hearts. (A) Immunofluorescence staining of Nogo-A/B (red) and membrane glycoproteins with wheat germ agglutinin (WGA; green) in murine and human hearts. The inset shows the staining for Nogo-A/B in NogoA/B-deficient heart sections, as a negative control. The nuclei were counterstained with DAPI (blue). Scale bar: $50 \mu \mathrm{m}$. (B) Western blot analysis and quantification of Nogo-A and Nogo-B expression in lysates of hearts of transverse aortic constriction-operated (TAC-operated) and sham-operated WT mice at indicated time points. Nogo-A/B-deficient hearts $\left(\mathrm{Ng}^{-1}\right)$ were used as a negative control. CAPDH was used as loading control. (C) Sections of hearts from sham- and TAC-operated WT mice at indicated time points were stained for Nogo-A/B (red), WGA (green), and DAPI (blue). Cardiomyocytes positive for Nogo-A/B were counted in sections from the base, center, and apex of the hearts and are expressed as a percentage of the total number of cardiomyocytes counted per heart section, as shown below images. $n=5$ /group. Scale bar: $100 \mu \mathrm{m}$. (D) Serial sections of sham-operated hearts and hearts 3 days after TAC were stained with anti-Nogo-A antibody and anti-Nogo-A/B antibodies. Nogo-A staining localized exclusively in cardiomyocytes and was not observed in the vasculature or fibroblasts. Scale bar: $50 \mu \mathrm{m}$. (E) Western blot analysis of Nogo-A and Nogo-B in cardiomyocytes isolated from sham- and TAC-operated WT hearts at indicated time points. Lysates prepared from the whole WT hearts were used as positive control, whereas lysate from Nogo-A/B-deficient heart was used as negative control. GAPDH was used as loading control. Data are expressed as mean $\pm \mathrm{SEM} .{ }^{*} P<0.05,{ }^{* *} P<0.01,{ }^{* * *} P<0.001(\mathbf{B}$ and $\mathbf{C}$ ), based on 1-way ANOVA followed by Tukey's multiple comparison test.

The loss of Nogo-A/B protected mice from pressure overload-induced cardiac hypertrophy and dysfunction. Recently, we reported that mice lacking Nogo-A/B are markedly hypotensive ( $25 \mathrm{mmHg})$ compared with WT mice, with no difference in heart rate (18), suggesting a reduced pressure load on the heart under physiological conditions. In mice, cardiomyocyte proliferation contributes to the postnatal growth of the heart for the first week after birth $(23,24)$; thereafter, heart growth is mainly accounted for by the increase in cardiomyocyte volume (25-27) in response to hemodynamic stimuli. Whereas at 4 weeks of age, the heart weight/tibia length ratios of WT and Nogo-A/B-deficient mice were similar, the hearts of 5-, 6-, 7-, and 8-week-old Nogo-A/B deficient mice were significantly smaller than those of WT littermates (Figure 2, A and B, sham panels), suggesting that the loss of Nogo-A/B did not affect cardiomyocyte hyperplasia, but rather postnatal hypertrophy. 
A

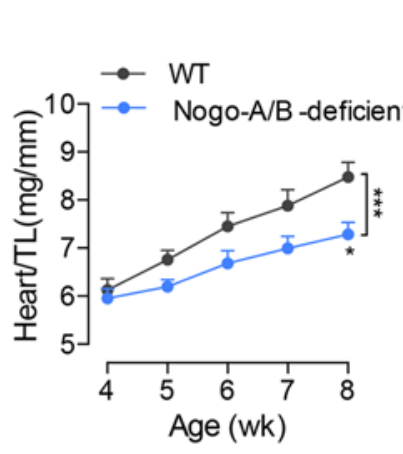

B

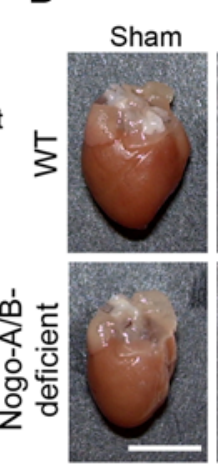

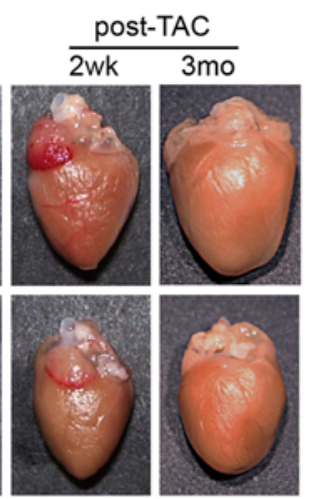

$\mathbf{F}$
C

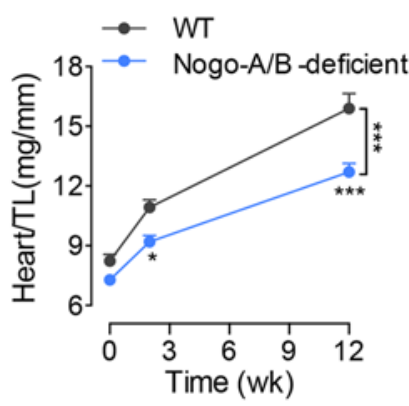

D
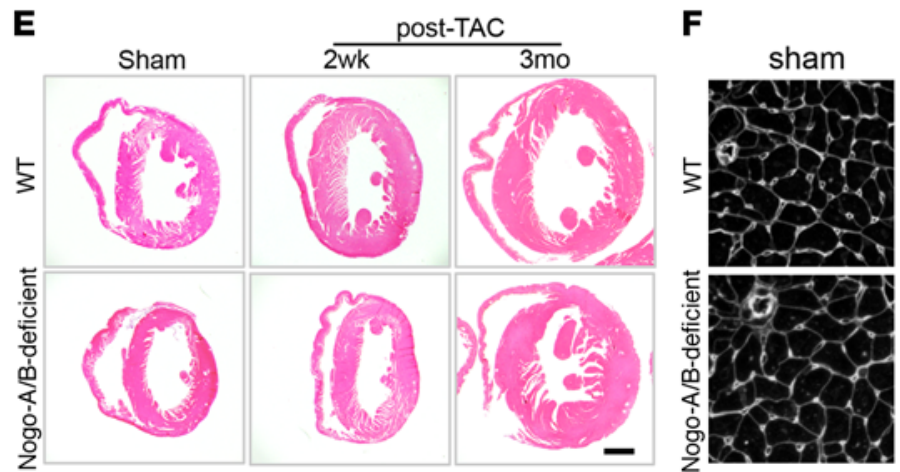

G
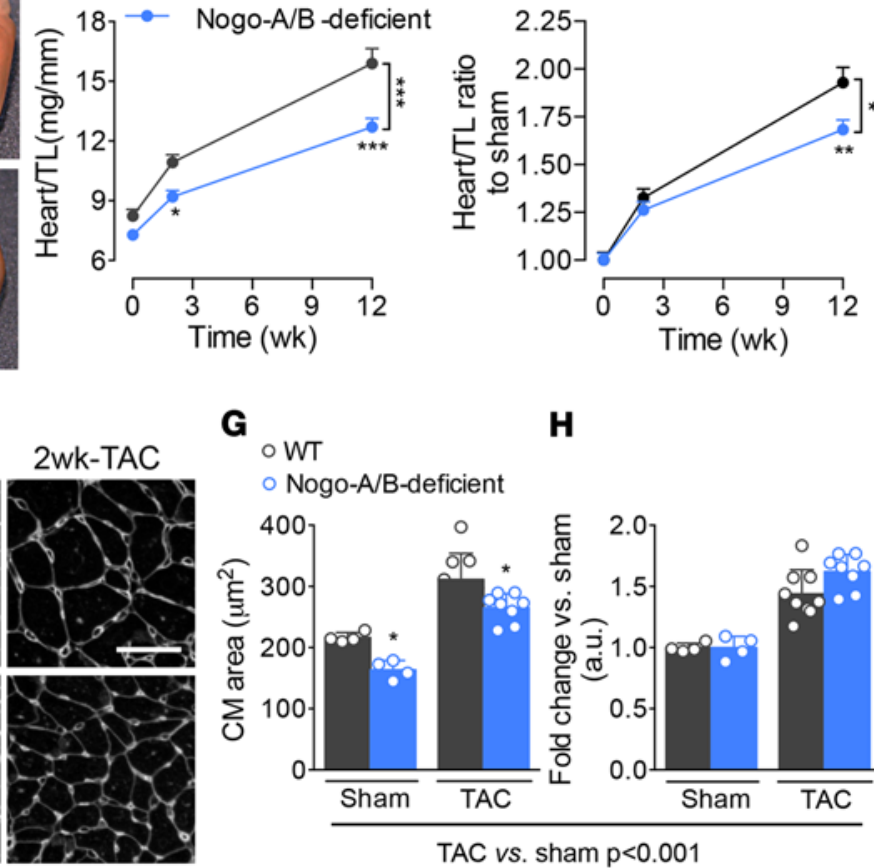

H
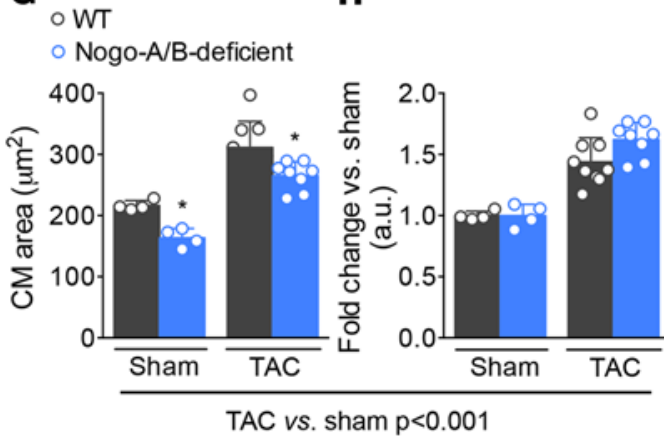

Figure 2. Nogo-A/B-deficient mice were protected from pathological hypertrophy induced by chronic pressure overload. (A) Heart weight/tibia length ratios (heart/TL) of WT and Nogo-A/B-deficient mice at 4, 5, 6, 7, and 8 weeks of age. $n \geq 6$ /group. (B) Representative images of WT and Nogo-A/B-deficient hearts of sham- and transverse aortic constriction-operated (TAC-operated) mice at indicated time points. Scale bar: $5 \mathrm{~mm}$. (C) Analysis of heart/TL ratios for TAC- and sham-operated WT and Nogo-A/B-deficient mice at indicated time points. $n \geq 8 /$ group. (D) Heart/TL ratios for sham- and TAC-operated WT and Nogo-A/B-deficient mice expressed as fold increase over the respective sham-operated groups. $n \geq 8 /$ group. (E) Hematoxylin and eosin staining of cross sections of hearts of TAC- or sham-operated WT and Nogo-A/B-deficient mice at indicated time points. Scale bar: 1 mm. (F) Immunofluorescence staining with WCA of cardiac cross sections from sham-operated WT and Nogo-A/B-deficient mice or mice 2 weeks after TAC. Scale bar: 50 $\mu \mathrm{m}$. (C) Absolute and (H) relative-to-sham quantification of cardiomyocyte cross-sectional area in sham-operated WT and Nogo-A/B-deficient hearts and hearts 2 weeks after TAC. $n=4$ sham-operated and $n=8$ banded-heart. Data are expressed as mean \pm SEM. ${ }^{*} P<0.05,{ }^{* *} P<0.01,{ }^{* * *} P<0.001$ compared with WT at the indicated time points or treatments. Statistical significance was determined by (C) 1-way ANOVA followed by Tukey's multiple comparison test or (A, C, and $\mathbf{D})$ 2-way ANOVA followed by Tukey's multiple comparison test.

Next, we investigated the role of Nogo in pathological hypertrophy of the heart exposed to hemodynamic stress. Following TAC, WT mice developed sustained cardiac hypertrophy at 2 weeks, reaching a 2-fold increase in heart size at 3 months (Figure 2, B-D), with marked dilation of the left ventricle (Figure $2 \mathrm{E}$ ). The heart weight/tibia length ratios of Nogo-A/B-deficient mice were significantly reduced in shamoperated mice and in mice at 2 weeks and 3 months after TAC, compared with WT mice (Figure 2C). However, the fold increase in heart weight/tibia length ratios of TAC- versus sham-operated hearts (Figure 2D) demonstrated that the loss of Nogo-A/B did not alter the magnitude of the hypertrophic response to pressure overload in the initial phase but protected the hearts from developing dilated cardiomyopathy at 3 months after TAC (Figure 2, D and E). To further corroborate the presence of hypertrophic remodeling in the absence of Nogo-A/B, we assessed the cardiomyocyte cross-sectional area, known to increase during pressure overload stress. The area of Nogo-A/B-deficient cardiomyocytes was significantly reduced in shamoperated mice and in mice 2 weeks after TAC, compared with WT mice (Figure 2, F and G), data supported by the expression levels of hypertrophy-associated genes at 2 weeks after TAC, including atrial natriuretic peptide (Nppa), brain natriuretic peptide (Nppb), and $\beta$-myosin heavy chain ( $M y h 7$; Supplemental Figure 3). However, in agreement with the heart weight/tibia length ratios, the magnitude of increase in cardiomyocyte area was the same in TAC and sham-operated hearts at 2 weeks after TAC (Figure 2H). Interestingly, vascularity of WT and Nogo-A/B-deficient mice, expressed as a ratio of the number of capillaries per the number of cardiomyocytes, was not different between the two groups in sham- and TAC-operated mice (Supplemental Figure 1, B and C). This suggests that, whereas the lack of Nogo-A/B does not alter the initial 


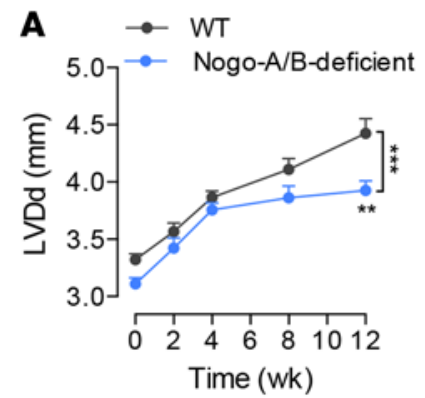

B

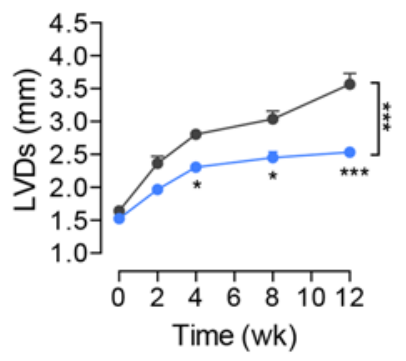

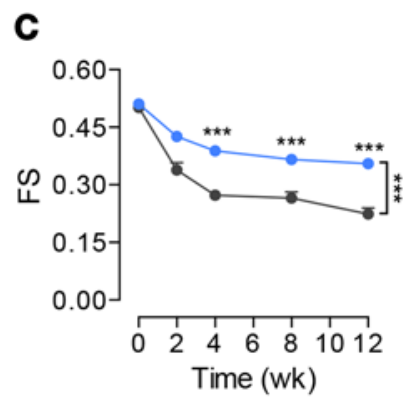

D
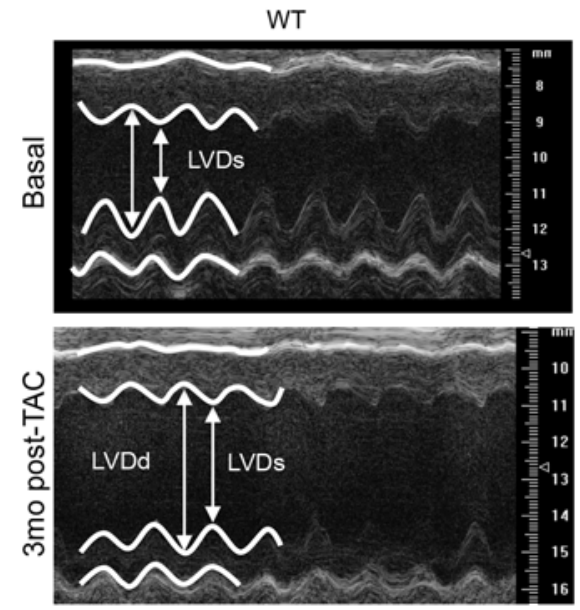
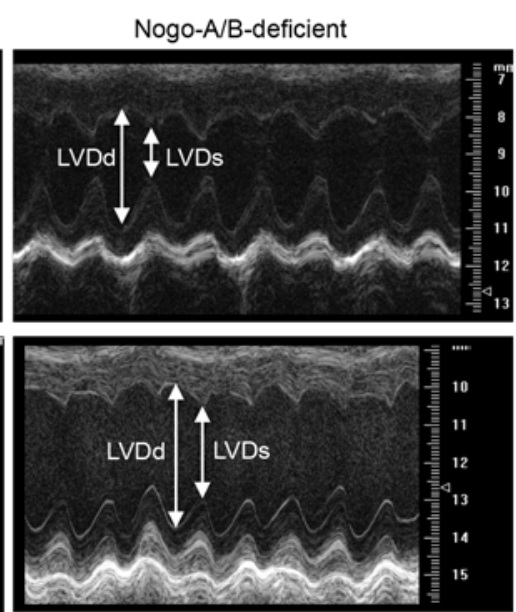

Figure 3. Loss of Nogo-A/B protected mice from left ventricle dilation and systolic contractile dysfunction following chronic pressure overload. Serial echocardiographic analysis of WT and Nogo-A/B-deficient mice before and after transverse aortic constriction (TAC) surgery. (A) Left ventricle (LV) end-diastolic diameter (LVDd), (B) LV end systolic (LVDs) diameter, and (C) fractional shortening (FS) were measured at the indicated time points after TAC. $n \geq$ 15/group. (D) Representative images of 2-dimensional guided M-mode echocardiography of the LV of WT and Nogo-A/B-deficient mice at baseline and 3 months after TAC. Data are expressed as mean \pm SEM. ${ }^{*} P<0.05$, ${ }^{* *} P<0.01,{ }^{* * *} P<0.001$ compared with WT. Statistical significance was determined by 2-way ANOVA followed by Tukey's multiple comparison test.

adaptive hypertrophic response to pressure overload, it reduces the magnitude of cardiac hypertrophy and left ventricle dilation in response to long-term hemodynamic stress, thus preserving the heart morphometry.

To assess whether these morphometric changes mirrored functional differences, we performed transthoracic echocardiography at different time points after TAC. Serial echocardiographic analysis revealed progressive cardiac dysfunction in WT banded hearts, with increasing left ventricle end-diastolic dimension (LVDd) and end-systolic dimension (LVDs) and a dramatic reduction in fractional shortening (Figure 3). In contrast, Nogo-A/B-deficient hearts were protected from failure for up to 3 months after TAC, preserving LVDd and LVDs, compared with WT hearts (Figure 3), suggesting that the lack of Nogo-A/B efficiently protects the heart from failing following chronic pressure overload.

Nogo-B promotes plasma and inflammatory cell extravasation in pressure-overloaded hearts. A variety of studies have implied an important role of the inflammatory response in the pathogenesis of heart failure $(28,29)$. Although inflammation is often considered from the perspective of leukocyte and lymphocyte infiltrating the tissue, the endothelium actively participates in and regulates the inflammatory processes. In pressure-overloaded hearts, tension is distributed throughout the whole coronary artery tree, and the vascular wall becomes the primary source of proinflammatory signals that lead to the initiation of inflammation and myocardial damage $(6,30-32)$. Recently, we reported that, in the absence of Nogo-B, the endothelium is more resistant to extravasation of inflammatory cells, despite unchanged levels of adhesion molecules (17). Indeed, primary cultures of endothelial cells isolated from Nogo-A/B-deficient lungs showed higher resistance to the passage of current than WT cells, providing a quantitative measurement of the increase in barrier function in the absence of Nogo-B (Figure 4A). Thus, to confirm the role of Nogo-B in endothelial barrier function in pressure-overloaded hearts, we injected banded and nonbanded mice with the albumin-binding dye Evans Blue 24 hours after surgery. Whereas extravasation of albumin-bound Evans 
A

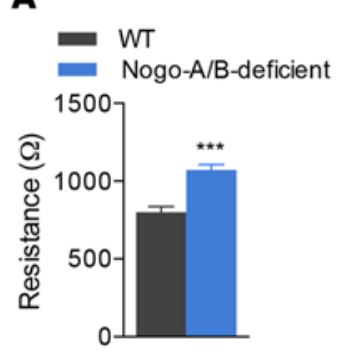

B

- WT

- Nogo-A/B -deficient

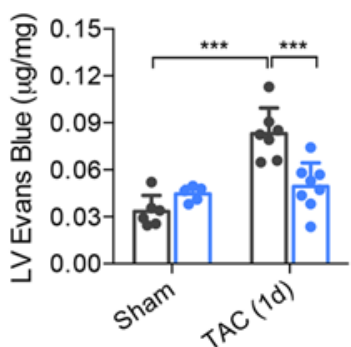

C

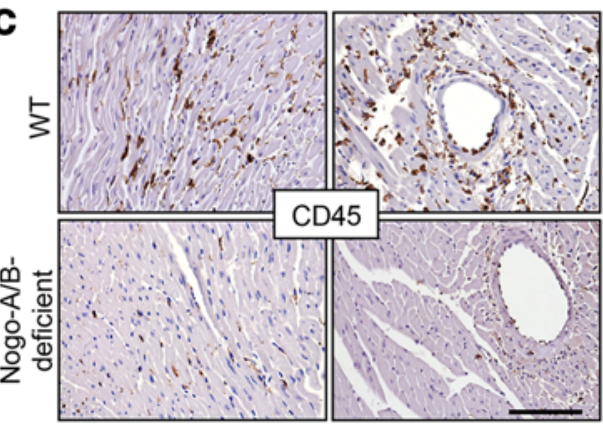

D

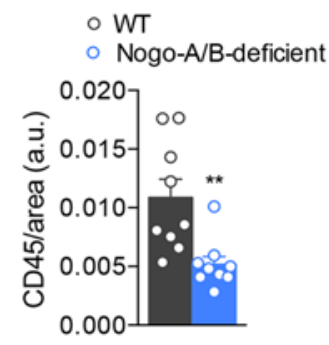

E 2wk post-TAC 3mo post-TAC $\mathbf{F}$

G

H

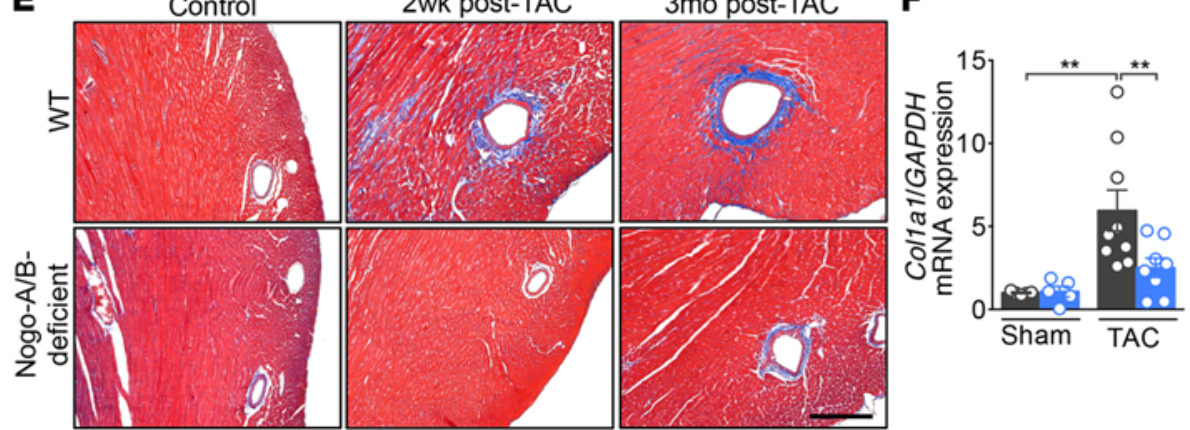

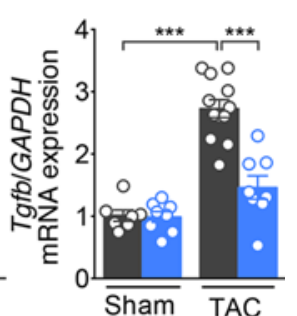

Figure 4. Nogo-A/B-deficient mice were resistant to myocardial permeability, inflammation, and fibrosis. (A) Assessment of the endothelial barrier functions by measuring the resistance $(\Omega)$ of primary cultures of WT and Nogo-A/B-deficient endothelial cells using the Electric Cell-Substrate Impedance Sensing System. $n=14$ replicates from 4 independent endothelial cell isolations/group. (B) Myocardial permeability was assessed by quantifying the extravasation of Evans Blue dye into the hearts of sham- or transverse aortic constriction-operated (TAC-operated) WT and Nogo-A/B-deficient mice and was expressed as $\mu \mathrm{g} / \mathrm{mg}$ dry heart weight. $n \geq 6$ /group. (C) Images and (D) quantification of immunohistochemical staining of CD45 in heart sections from WT and Nogo-A/Bdeficient mice at 3 days after TAC. Scale bar: $100 \mu \mathrm{m}$. $n \geq 9$ 9/group. (E) Masson's trichrome staining of cardiac fibrosis in TAC- or sham-operated WT and NogoA/B-deficient mice at indicated time points. Scale bar: $200 \mu \mathrm{m}$. Real-time PCR analysis of expression of fibrosis-related genes (F) collagen type I $\alpha 1$ (Col1a1), (G) collagen type $3 \alpha 1$ (Col3a1), and (H) Tgfb in sham or 2 weeks banded WT and Nogo-A/B-deficient hearts. Gapdh served as internal control. Data were normalized to sham group. $n \geq 9$ /group. Data are expressed as mean $\pm S E M$. ${ }^{* *} P<0.01$, ${ }^{* *} P<0.001$ compared with WT or as otherwise indicated. Statistical significance was determined by unpaired $t$ test (A and $\mathbf{D}$ ) or 1-way ANOVA followed by Tukey's multiple comparison test (B and F-H).

Blue was markedly increased in banded WT hearts compared with sham-operated hearts, it was attenuated in banded Nogo-A/B-deficient hearts (Figure 4B), suggesting that Nogo-B plays an important role in the initial phase of inflammation following hemodynamic stress.

Perivascular inflammation and fibrosis are intimately linked in hypertensive disease and contribute to myocardial and arterial stiffness, compromising the pumping capacity of the heart and oxygen diffusion to the hypertrophied myocytes. Nogo-A/B-deficient mice were resistant to pressure overload-induced perivascular and interstitial inflammation (Figure 4, C and D, and Supplemental Figure 4) and fibrosis (Figure 4E) at 3 days and 2 weeks after TAC, respectively. These findings were confirmed by reduced expression of myocardial fibrogenesis-related genes, including collagen 1A1 (Colla1), collagen 3A1 (Col3a1), and Tgfb (Figure 4, F-H).

Endothelial Nogo-B controls the progression of maladaptive left ventricle remodeling and systolic dysfunction induced by chronic pressure overload. To validate the role of endothelial Nogo-B in the pathogenesis of heart failure, we generated mice lacking Nogo-B specifically in the endothelial cells (18) by crossbreeding floxed Nogo-A/B mice with VE-cadherin-Cre ${ }^{\mathrm{ERT} 2}$ mice (33). Real-time PCR analysis of Nogo-B in murine lung endothelial cells (MLECs) confirmed efficiency of excision greater than $85 \%$ (18). Furthermore, Nogo-B was undetectable by immunofluorescence staining in the endothelium of coronary arteries of ECNogo-A/B-deficient mice (Figure 5A). Similar to mice systemically lacking Nogo-A/B, EC-Nogo-A/Bdeficient mice were resistant to pressure overload-driven vascular permeability (Figure $5 \mathrm{~B}$ ) and inflammation (Figure 5, C and D) compared with control (Nogo-A/B ${ }^{\mathrm{f} / \mathrm{f}}$ ) littermates.

Serial echocardiographic measurements revealed preserved cardiac function in EC-Nogo-A/B-deficient mice compared with Nogo-A/B ${ }^{\mathrm{f} / \mathrm{f}}$ mice, which showed progressive cardiac dysfunction $(0.38 \pm 0.01$ $\mathrm{mm}$ vs. $0.23 \pm 0.01 \mathrm{~mm}$ ) at 3 months after TAC (Figure 5, E-G). Collectively, our data suggest that endothelial Nogo-B plays a crucial role in the onset of heart failure induced by chronic hemodynamic stress 


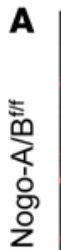
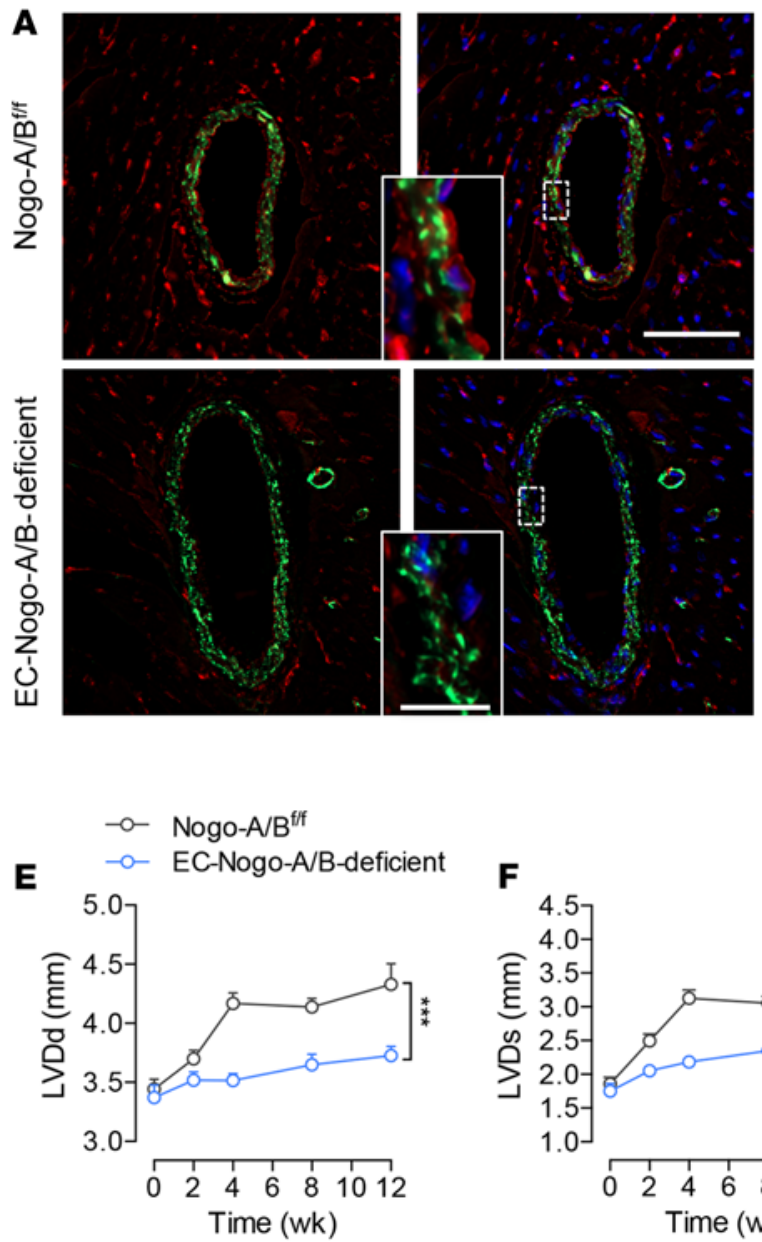

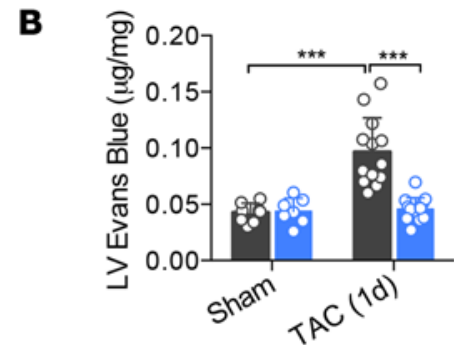

c

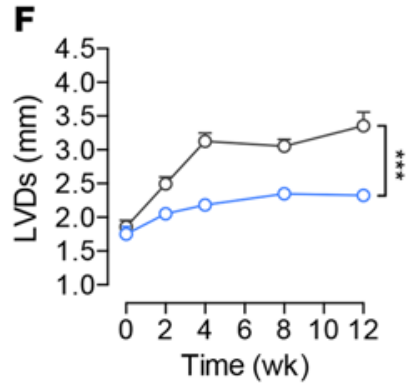

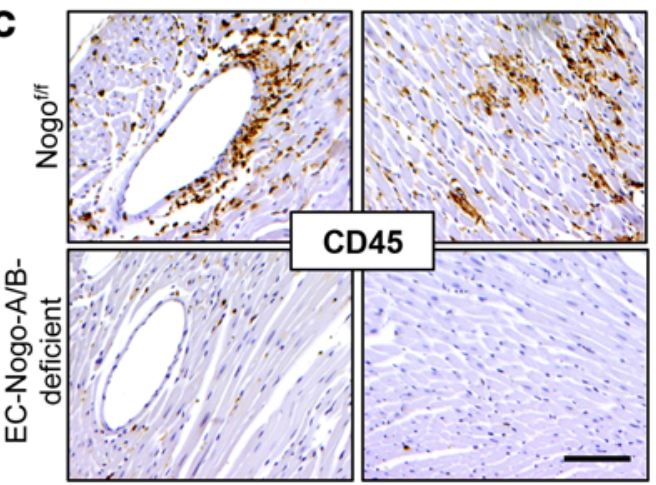

G
D

oNogo-A/B ${ }^{\text {ff }}$ -EC-Nogo-A/B-deficient

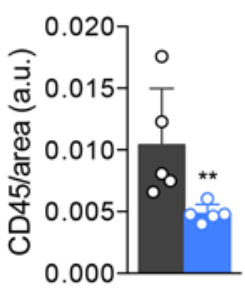

Figure 5. Endothelial Nogo-B controls the progression of maladaptive left ventricle remodeling and systolic dysfunction induced by pressure overload. (A) Immunofluorescence staining of Nogo-A/B (red) and $\alpha$-smooth muscle actin ( $\alpha$-SMA; green) in sections of heart tissue from Nogo-A/B ${ }^{f / f}$ and EC-Nogo-A/B-deficient mice. Scale bar: $50 \mu \mathrm{m}$. Inset, higher-magnification view of outlined area. Scale bar: $10 \mu \mathrm{m}$. (B) Myocardial permeability assessed by Evans Blue extravasation in sham- and transverse aortic constriction-operated (TAC-operated) Nogo-A/B ${ }^{\mathrm{f} / \mathrm{f}}$ and EC-Nogo-A/B-deficient mice 24 hours after surgery. $n \geq 7 /$ group. (C) Immunohistochemical staining and (D) quantification of CD45 in myocardial sections from Nogo-A/B ${ }^{f / f}$ and EC-Nogo-A/Bdeficient mice at 3 days after TAC. $n \geq 5 /$ group. Scale bar: $100 \mu \mathrm{m}$. (E-C) Progressive left ventricle dysfunction of Nogo-A/B/f and EC-Nogo-A/B-deficient mice at baseline and 3 months after TAC. $n \geq 15$ /group. LVDd, left ventricle end-diastolic diameter; LVDs, left ventricle end systolic diameter; FS, fractional shortening. Data are expressed as mean \pm SEM. ${ }^{* *} P<0.01,{ }^{* * *} P<0.001$. Statistical significance was determined by (B) 1-way ANOVA followed by Tukey's multiple comparison test, (D) unpaired $t$ test, or (E-G) 2-way ANOVA followed by Tukey's multiple comparison test.

Nogo-B controls endothelial-derived sphingolipids and S1P to affect pathological cardiac hypertrophy in a paracrine fashion. SPT is the first and rate-limiting enzyme of de novo sphingolipid biosynthesis (34-36). Recently, we discovered that Nogo-B regulates the production of endothelial-derived sphingolipids, and particularly S1P, by inhibiting SPT activity (18). Hence, mice lacking Nogo-A/B show hyperactivation of the S1P/ $\mathrm{S}_{1} \mathrm{P}_{1}$ axis and are resistant to inflammation (17) and hypertension (18). With this knowledge in mind, we hypothesized that the sphingolipid de novo pathway exerts a protective effect during pathological cardiac hypertrophy following chronic pressure overload. Thus, we first assessed SPT activity in the hearts of WT and Nogo-A/B-deficient mice following TAC or sham surgery. Pressure overload significantly increased SPT activity in WT hearts at 3 days after TAC. In the absence of Nogo-A/B, SPT activity was markedly increased in sham-operated hearts and in hearts 3 days after TAC compared with WT hearts (Figure 6A), suggesting that pressure overload induces activation of SPT and sphingolipid de novo biosynthesis, exerting protective effects on the heart.

During pressure overload, the endothelium plays an active role in initiation of the inflammatory process leading to myocardial damage (3-6). Given that Nogo-B is highly expressed in the coronary artery tree but not in cardiomyocytes (Figure 1), we focused on modulation of the sphingolipid de novo pathway in the 
A

$$
\text { - Nogo-A/B -deficient }
$$

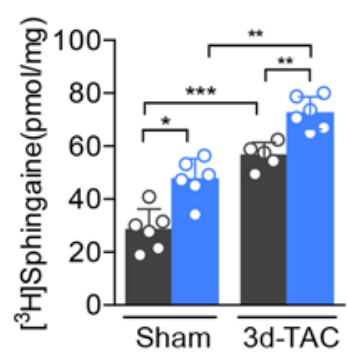

B

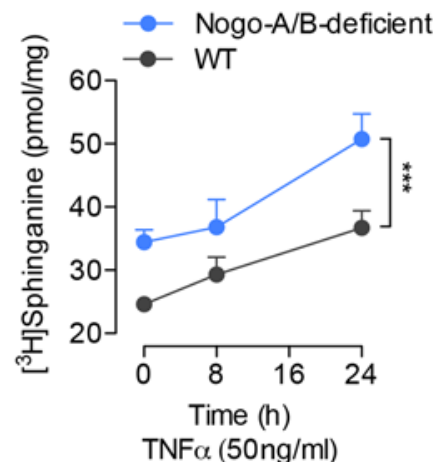

C

\section{HUVEC}

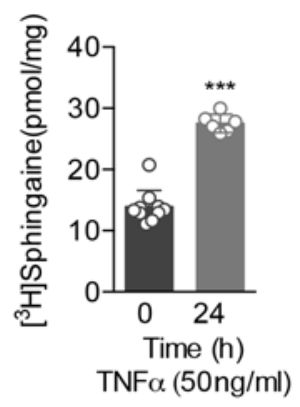

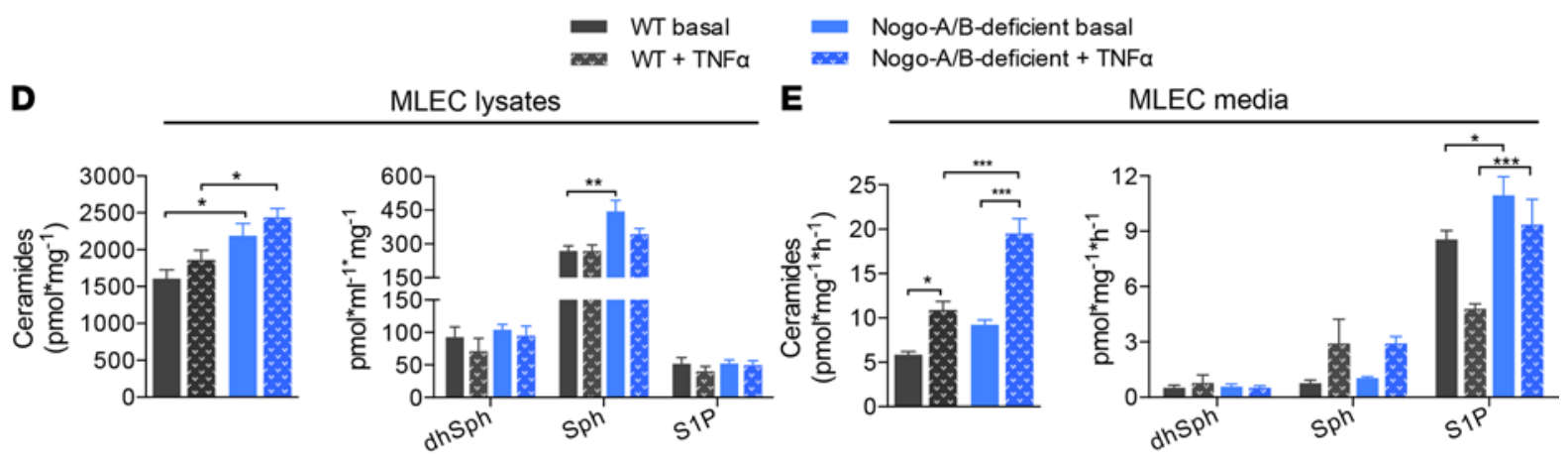

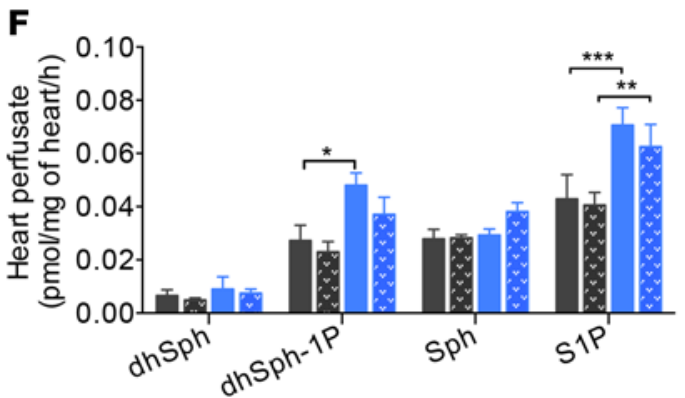

Figure 6. Endothelial Nogo-B regulates de novo synthesis of sphingolipid during chronic inflammation. (A) SPT activity assay was performed in heart microsomes from WT and Nogo-A/B-deficient mice at 3 days after transverse aortic constriction (TAC) or sham operation. SPT activity was measured using [ $\left.{ }^{3} \mathrm{H}\right]$-serine as a substrate, followed by TLC separation of sphinganine, a downstream product of SPT. $n \geq 5$ /group. (B) Assessment of SPT activity in WT and Nogo-A/B-deficient mouse lung endothelial cells (MLEC) treated with TNF- $\alpha(50 \mu \mathrm{g} / \mathrm{ml})$ or vehicle at indicated time points. $n \geq 11$ replicates from 5 to 6 independent MLEC isolations. (C) SPT activity assay of human umbilical vein endothelial cells (HUVEC) treated for 24 hours with TNF- $\alpha(50 \mu \mathrm{g} / \mathrm{ml}$ ) or vehicle. $n=10$ replicates/group. (D and E) Total ceramide and dihydrosphingosine (dhSph), sphingosine (Sph), sphingosine-1-phosphate (S1P) levels in WT and Nogo-A/B-deficient (D) MLECs and (E) culture medium. $n \geq 12$ replicates from 4 independent isolations of MLECs/group. (F) Levels of dhSph, dhSph-1-phosphate (dhSph-1P), Sph, and S1P in the perfusates of WT and Nogo-A/B-deficient hearts of sham-operated mice or mice 3 days after TAC. $n \geq 9 /$ group. Data are expressed as mean \pm SEM. ${ }^{*} P<0.05,{ }^{* *} P<0.01,{ }^{* * *} P<$ 0.001. Statistical significance was determined by (A and $\mathbf{D}-\mathbf{F})$ 1-way ANOVA followed by Tukey's multiple comparison test, (B) 2-way ANOVA, or (C) unpaired $t$ test.

inflamed endothelium. TNF- $\alpha$ induced a time-dependent activation of SPT in primary cultures of WT endothelial cells isolated from MLECs (Figure 6B) as well as in cultured human umbilical vein endothelial cells (HUVECs) (Figure 6C). Furthermore, in the absence of Nogo-B, SPT activity was significantly increased compared with WT MLECs before, and at different time points after, TNF- $\alpha$ activation (Figure 6B).

Cultured endothelial cells rapidly secrete S1P (37) through specialized transporters (38-40). Therefore, we measured sphingolipid and S1P levels in cell lysates and culture medium of MLECs. In agreement with previous studies (41), we observed no differences in ceramide, dihydrosphingosine (dhSph), sphingosine (Sph), and S1P levels following TNF- $\alpha$ stimulation, although cells lacking Nogo-B maintained higher sphingolipid levels than WT cells, in the basal as well as the activated state (Figure 6D). Interestingly, TNF- $\alpha$ induced a marked increase in ceramide, Sph, and S1P in the medium of inflamed endothelial cells, with significantly higher levels in the medium of Nogo-A/B-deficient cells (Figure 6E). 
A

TAC or sham surgery Time (d) -2 \begin{tabular}{cccc}
$\boldsymbol{- 1}$ & 0 & 1 & 2 \\
\hline myriocin & 0.3 & $\mathbf{m g} / \mathrm{kg}$
\end{tabular}

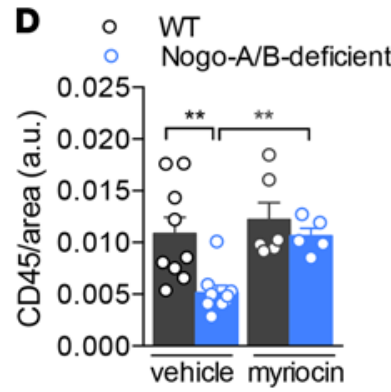

H

TAC or sham surgery Time (d) \begin{tabular}{rrrrr}
-1 & 0 & 1 & 2 & 3 \\
\hline $\begin{array}{l}\mathbf{4} \\
\mathbf{4}\end{array}$ & $\mathbf{4}$ & $\mathbf{4}$ & $\mathbf{4}$ & $\mathbf{4}$ \\
SEW 2871 & $3 \mathrm{mg} / \mathrm{kg}$
\end{tabular}
B - $w T$

- Nogo-A/B-deficient

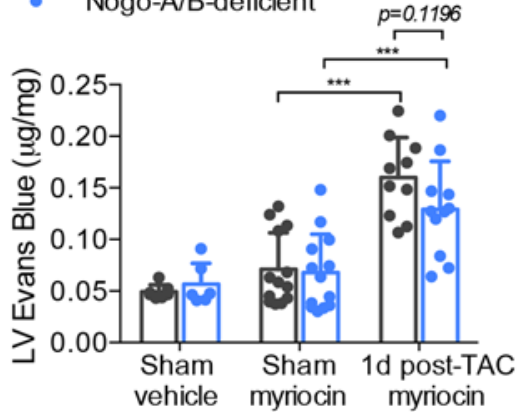

C

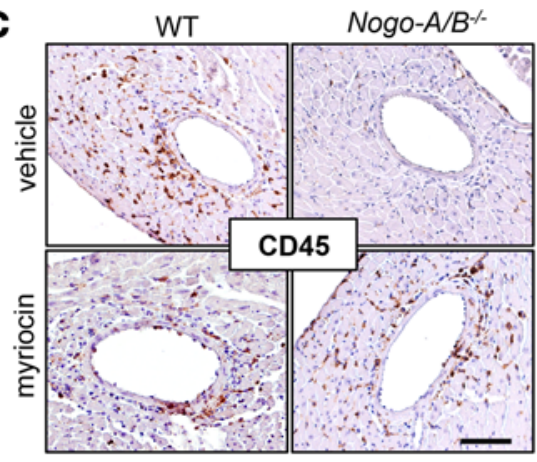

E

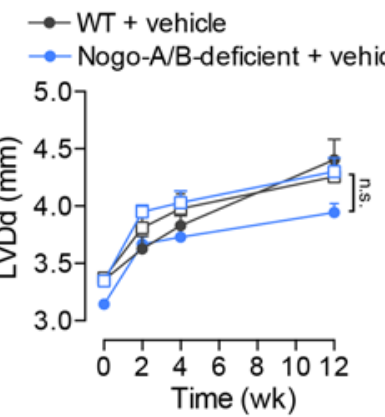

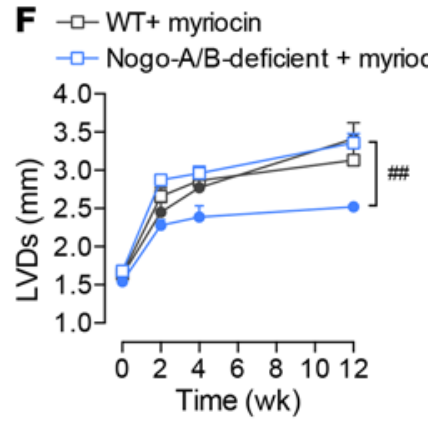

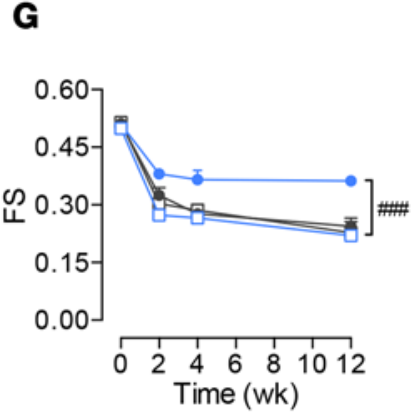

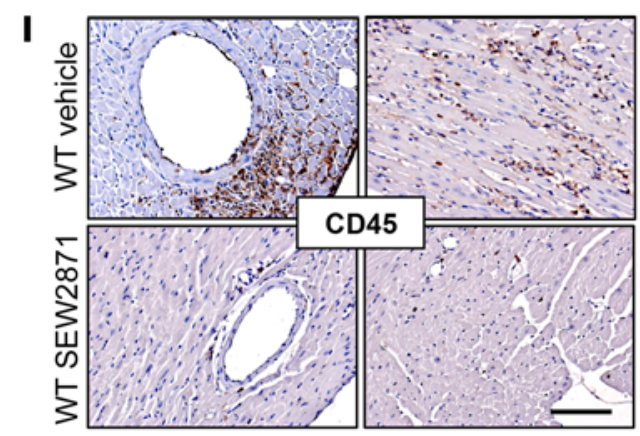
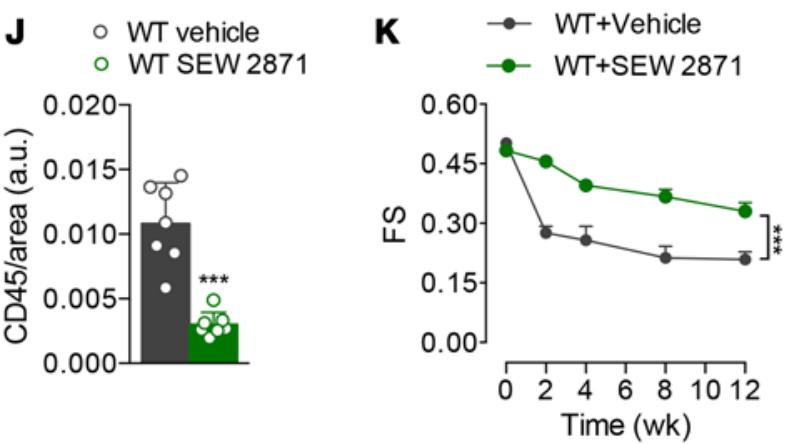

$\mathbf{L}$

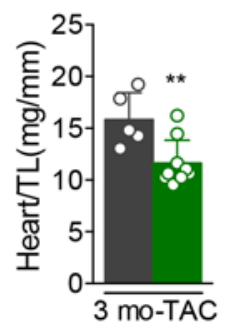

Figure 7. Endothelial Nogo-B regulates de novo synthesis of sphingolipid to affect cardiac pathogenesis through SPT/S1P signaling. (A) Experimental design related to B and C: WT and Nogo-A/B-deficient mice were i.p. injected with a selective inhibitor of SPT, myriocin (0.3 mg/kg), or vehicle (0.4\% fatty acid-free BSA) at the indicated time points. (B) Myocardial permeability assessed 24 hours after surgery by quantifying Evans Blue extravasated into sham-operated or banded WT and Nogo-A/B-deficient hearts. $n \geq 6$ /group. (C) Inflammation was assessed at 3 days after surgery in WT and Nogo-A/B-deficient mice treated with myriocin or vehicle by immunostaining of CD45 in heart sections and (D) quantification. Scale bar: $100 \mu \mathrm{m} . n \geq 6$ mice/group. Serial echocardiographic analyses of transverse aortic constriction-operated (TAC-operated) WT and Nogo-A/B-deficient mice treated with myriocin $(0.3 \mathrm{mg} / \mathrm{kg})$ or vehicle. The parameters measured were (E) left ventricle (LV) end-diastolic diameter (LVDd), (F) LV end systolic diameter (LVDs), and (C) LV fractional shortening (FS). $n \geq 7$ mice/vehicle group; $n=$ 7-11 mice/myriocin group. (H) Experimental design: WT mice were i.p. injected with an agonist of S1P, receptor, SEW2871 (3 mg/kg), or vehicle (DMSO) as shown. (I) Images and (J) quantification of immunohistochemical staining of CD45 in sections of hearts from SEW2871- and vehicle-treated mice 3 days after TAC. $n \geq 7$. Scale bar: $100 \mu \mathrm{m}$. (K) Serial echocardiographic analyses of TAC-operated mice treated with SEW2871 (3 mg/kg) or vehicle as indicated in H. $n=5$ vehicle-treated WT mice; $n=9$ SEW2871-treated mice. (L) Analysis of heart weight/tibia length ratios (heart/TL) of hearts from SEW2871- or vehicle-treated mice at 3 months after TAC. $n \geq 5$ /group. Data are expressed as mean \pm SEM. ${ }^{* *} P<0.01,{ }^{* * *} P<0.001,{ }^{*} P<0.05$, ${ }^{\# \#} P<0.001$ for myoricin- versus vehicle-treated Nogo-A/B-deficient mice. Statistical significance was evaluated with (B and D) 1-way ANOVA followed by Tukey's multiple comparison test, (E-G and K) 2-way ANOVA followed by Tukey's multiple comparison test, and ( $\mathbf{J}$ and $\mathbf{L}$ ) unpaired $t$ test. 
To corroborate these findings in vivo, hearts 3 days after TAC and control hearts were isolated and perfused for 30 minutes through the coronary vasculature with perfusion buffer (AfCS) containing phosphatase inhibitors, as previously described (42), and sphingolipids were measured in the buffer following perfusion. The rapid (30 $\mathrm{min}$ ) accumulation of S1P in the coronary vasculature perfusate is noteworthy and confirms the direct and active release of S1P by the endothelium in the heart ex vivo. The levels of S1P and dhSph-1-phosphate (dhSph-1-P) produced by the Nogo-A/B-deficient coronary endothelium were consistently higher than WT at both basal time points and those after TAC (Figure 6F). Under these experimental conditions, we observed no difference in the levels of ceramide species in the two groups (data not shown).

These findings, together with in vivo data from EC-Nogo-A/B-deficient mice, suggest that, following pressure overload, SPT activity in the coronary vasculature increases and removal of Nogo-B inhibition of SPT in the myocardial endothelium is cardioprotective, underlying the important role of vascular-derived sphingolipids on cardiac functions.

Myriocin inhibition of SPT restores myocardial inflammation and dysfunction in response to pressure overload in Nogo-A/B-deficient hearts. To validate the hyperactivation of the sphingolipid de novo pathway as the mechanism whereby Nogo-A/B-deficient mice were protected from pathological cardiac hypertrophy during chronic pressure overload, SPT activity was inhibited with myriocin 1 day before and on day 0 and day 2 after surgery, as shown in Figure 7A. Myriocin restored myocardial permeability and inflammation following pressure overload in Nogo-A/B-deficient mice (Figure 7, B-D) to WT levels, suggesting that the sphingolipid biosynthetic pathway protected the heart from inflammation during chronic hemodynamic stress. Furthermore, myriocin, administered only during the onset of pressure overload (Figure 7A), reestablished cardiac dysfunction in Nogo-A/B-deficient mice up to 3 months after TAC (Figure 7, E-G). Taken together, these findings suggest that Nogo-B-mediated inhibition of SPT plays an important role in cardiac dysfunction induced by pressure overload, because, in mice lacking Nogo-B, de novo synthesis of sphingolipids is increased and the animals are protected from heart failure during chronic pressure overload, effects that were abolished by myriocin.

To confirm the role of the $\mathrm{S} 1 \mathrm{P} / \mathrm{S}_{1} \mathrm{P}_{1}$ signaling pathway in the cardioprotective phenotype observed in mice lacking Nogo, WT mice were treated with SEW2871, an agonist of $\mathrm{S}_{1} \mathrm{P}_{1}$ receptor, or vehicle at different time points after TAC (Figure 7, H-L). Perivascular and interstitial inflammation triggered by high intraventricular pressure was attenuated in WT mice treated with SEW2871 (Figure 7, I and J). Echocardiographic analysis showed that SEW2871 protected WT hearts from hypertrophy and failure for up to 3 months after TAC, compared with vehicle-treated mice (Figure 7, $\mathrm{K}$ and $\mathrm{L}$ ). These findings suggest that targeting $\mathrm{S}_{1} \mathrm{P}_{1}$ signaling in pressure-overloaded hearts reduces acute inflammation and protects hearts from long-term cardiac dysfunction.

\section{Discussion}

In this study, we demonstrated that sphingolipids produced by endothelium, particularly S1P, play an important role in the pathogenesis of heart failure by controlling myocardial inflammation and fibrosis in response to high pressure and by regulating the remodeling and function of hearts exposed to chronic pressure overload. We also showed that Nogo-B, expressed in the coronary vasculature and specifically in the endothelium, negatively regulates local sphingolipid production to affect vascular and heart function during chronic pressure overload.

Alteration of sphingolipid biology has recently been correlated with various forms of cardiovascular disease, including atherosclerotic heart disease, cardiomyopathy, heart failure, and hypertension (43-46), suggesting a potential role for sphingolipids in these pathological conditions. An interesting study by Argraves and colleagues showed an inverse relationship between the levels of S1P in the plasma HDL fraction and the occurrence of ischemic heart disease, highlighting the role of S1P in mediating, at least in part, HDL-mediated cardioprotective effects (47). However, whether the de novo biosynthesis of sphingolipids is altered and what the biological significance is of different sphingolipid levels in specific cell types or in plasma during pathological cardiac hypertrophy are still unknown. Our findings showed marked upregulation of SPT activity in myocardial microsomes following pressure overload, with higher magnitude in Nogo-A/B-deficient mice. Furthermore, myriocin treatment reestablished myocardial permeability, inflammation, and failure in Nogo-A/B-deficient hearts, corroborating the protective role of sphingolipid de novo biosynthesis in the pathogenesis of heart failure. The importance of de novo sphingolipid biosynthesis in cardiac homeostasis is also demonstrated by a study by Lee and colleagues, who showed that mice lacking SPT activity in cardiomyocytes developed dilated cardiomyopathy (48). 
Mechanistically, Nogo-B binds to and inhibits SPT (18), thereby controlling local sphingolipid and $\mathrm{S} 1 \mathrm{P}$ production, which preserves blood flow through activation of the $\mathrm{S}_{1} \mathrm{P}_{1} / \mathrm{S}_{1} \mathrm{P}_{3} / \mathrm{eNOS}$ pathway (18), enhances endothelial barrier functions through $\mathrm{S}_{1} \mathrm{P}_{1}$-Rac activation $(12,49)$, and exerts antiinflammatory effects (50-52). Furthermore, recent findings identified protective functions of S1P in ischemia/reperfusion injury in several organs, including the heart $(11,53-55)$.

The increase of pressure in the ascending aorta, such as that seen with hypertension, is also distributed throughout the coronary arteries and has been correlated directly with myocardial permeability and fibrosis $(6,56,57)$. Thus, maintenance of endothelial-barrier integrity may limit deleterious inflammation of hearts challenged by high pressure.

Analysis of the coronary artery perfusate of hearts ex vivo represented a direct measurement of levels of sphingolipids and S1P produced by the myocardial vasculature. Our data showed a significant increase in S1P levels in the perfusates of Nogo-A/B-deficient hearts, in both sham- and TAC-operated mice, compared with WT mice. This observation supports a regulatory function of Nogo-B in de novo biosynthesis of sphingolipids, particularly S1P, in the coronary vasculature that affects heart function. Consistent with these findings, mice lacking Nogo-A/B, both systemically and specifically in the endothelium, were protected from myocardial permeability and inflammation, most likely through S1P-mediated enhancement of endothelial barrier function (12). Direct evidence was provided by marked reduction in inflammation, dysfunction, and hypertrophy of the hearts in response to pressure overload by the $\mathrm{S}_{1} \mathrm{P}_{1}$ receptor agonist SEW2871.

In the long term ( 3 months after surgery), Nogo-A/B-deficient mice were also protected from fibrosis, cardiac remodeling, and dysfunction, probably by different means: (a) indirectly, due to reduced myocardial permeability, inflammation, and fibrosis; (b) by hyperactivation of the S1P/eNOS/NO pathway (18) and, thus, increased endothelial-derived NO, which has been shown to have cardioprotective functions (58, 59); and (c) considering the vicinity of capillaries and myocytes, endothelial-derived S1P may target cardiomyocytes and exert a direct protective effect (60).

It is well established that myocardial inflammation triggers cardiomyocyte death, cardiac remodeling, and fibrosis, favoring the onset of heart failure. Several studies have suggested an important role for TNF- $\alpha$ in heart disease (61-63). However, the disappointing results of clinical trials of anti-TNF- $\alpha$ therapy for heart failure (64) led to reconsideration of the biological roles of TNF- $\alpha$ in the heart and suggested, perhaps, potential unknown protective effects of TNF- $\alpha$. Subsequently, independent lines of investigation demonstrated cardioprotective functions of TNF- $\alpha$ by different means(65-68). Among these, the study of Lecour and colleagues is noteworthy (65), in that it demonstrates a role of TNF- $\alpha$ in cardiac preconditioning through sphingolipid signaling. In particular, N-oleoylethanolamine, an inhibitor of acid ceramidase (69), attenuated TNF- $\alpha$-induced preconditioning, whereas cell-permeable $\mathrm{C} 2$ ceramide and S1P recapitulated the preconditioning cardioprotective phenotype, suggesting that TNF- $\alpha$ and sphingolipid signaling lie within the same pathway.

Here, we report that TNF- $\alpha$ stimulation of murine endothelial cells induces time-dependent upregulation of SPT activity, without a significant increase in total ceramide and Sph levels in the cells. The lack of change in intracellular ceramide levels following TNF- $\alpha$ stimulation is consistent with the findings of Slowik and colleagues that show that TNF did not induce production of measurable ceramide in cultured HUVECs and ceramide treatment failed to induce NF- $\mathrm{BB}$ activation (41). Strikingly, ceramide and $\mathrm{Sph}$, but not S1P, levels in the culture medium were markedly increased by TNF- $\alpha$, in agreement with the increased SPT activity in the inflamed endothelium. The decrease in S1P production by the inflamed WT endothelium could be due to altered Sph kinase-1 and -2 expression or activity and/or to changes in Sph phosphatase and lyase levels. Nevertheless, the intracellular and extracellular levels of sphingolipids and S1P were significantly increased in the absence of Nogo-B, under basal as well as TNF- $\alpha$-stimulated conditions, compared with those in WT MLECs, supporting the concept that endothelial-derived sphingolipids and S1P exert beneficial effects on cardiomyocyte function.

In vivo evidence supporting the importance of endothelial-derived sphingolipids and S1P in pathological cardiac hypertrophy was provided by cardioprotection of EC-Nogo-A/B-deficient mice from myocardial permeability, inflammation, and dysfunction induced by pressure overload. Over the past decades, multiple findings have supported the correlation between endothelial impairment and left ventricular mass and function (70-73). Because of the close proximity of capillaries and myocytes, our data suggest that, in addition to known bioactive molecules released by the endothelium, such as NO $(58,59,74-76)$, which influence myocardial performance, sphingolipids, and particularly S1P, may have important cardioprotective actions during hemodynamic stress. 
Despite growing knowledge of the molecular changes that occur during pathological heart hypertrophy, the specific mechanisms governing the transition of the heart from physiological to pathological hypertrophy remain elusive. Our findings suggest that sphingolipid de novo biosynthesis in myocardial tissue, and specifically in the endothelial cells, plays an important role in the onset of pathological cardiac hypertrophy following chronic pressure overload.

Under physiological conditions, Nogo-A/B-deficient hearts were significantly smaller than those of control littermates. Considering that, in the normal heart, Nogo-A and -B are not expressed in ventricular cardiomyocytes, and this size difference may be attributable, in part, to lower systemic blood pressure and, thus, reduced cardiac workload in Nogo-A/B-deficient than WT mice (18). Following chronic pressure overload (3 months after TAC), systemic or endothelial-specific lack of Nogo-A/B protected the hearts from developing the dilated cardiomyopathy and dysfunction observed in WT mice. It is noteworthy that, although the mass of Nogo-A/B-deficient hearts was consistently smaller than that of WT hearts under both sham and TAC conditions, the magnitude of cardiac hypertrophy was different, not at the initial phase of pressure overload but only at 3 months after TAC. This suggests that, in the absence of Nogo, the compensatory response of the heart to high pressure was preserved without manifestation of heart dilation. Interestingly, cardiac function was retained in Nogo-A/B-deficient mice after TAC at all time points.

Altogether, our findings suggest a critical role of endothelial Nogo-B in the transition from physiological to pathological cardiac hypertrophy. Nogo-B-dependent regulation of sphingolipid metabolism and signaling within the vasculature to control the onset of pathological hypertrophy is a concept that not only establishes a causative role of altered sphingolipid signaling in pathological cardiac hypertrophy, but also suggests that the sphingolipid de novo pathway may represent a potential pharmacological target for the treatment of this pathological condition.

\section{Methods}

Animals. Nogo-A/B-deficient (77) and Nogo-A/B floxed mice (Nogo-A/B ${ }^{\mathrm{f} / \mathrm{f}}$ ) were generated as previously described (18). Mice with endothelial cell-specific Nogo-A/B knockout (EC-Nogo-A/B-deficient mice) were obtained by crossing Nogo-A/ $\mathrm{B}^{\mathrm{f} / \mathrm{f}}$ mice with transgenic mice in which the VE-cadherin promoter drives expression of tamoxifen-responsive Cre (VE-Cad-Cre ${ }^{\text {ERT2 }}$ mice) (33). To assess the efficiency of Nogo-A/B excision in the endothelial cells following tamoxifen treatment, MLEC and thoracic aortic endothelial cell mRNAs were analyzed by real-time PCR as previously described (18).

Minimally invasive TAC. WT male C57BL/6J, Nogo-A/B-deficient, Nogo-A/B $\mathrm{B}^{\mathrm{f} / \mathrm{f}}$, and ECNogo-A/B-deficient mice (8-10 weeks of age) were subjected to TAC or sham operation (22). In brief, mice were anesthetized using a single i.p. injection of ketamine $(100 \mathrm{mg} / \mathrm{kg})$ and xylazine $(5 \mathrm{mg} / \mathrm{kg})$. A topical depilatory was applied to the chest, and the area was cleaned with Betadine and alcohol. A horizontal incision 0.5 to $0.8 \mathrm{~cm}$ in length was made at the second intercostal space. After retracting the thymus, the aortic arch was visualized with a dissecting scope (Zeiss Discovery.V8 Stereo) at low magnification. A 7-0 nylon ligature was tied between the innominate and left common carotid arteries with an overlying 27-gauge needle, which was then rapidly removed, leaving a discrete region of stenosis. In sham-operated animals the ligature was just tied loosely around the aorta.

Echocardiographic studies. Cardiac dimensions and function were analyzed by transthoracic echocardiography using a Vevo 770 Imaging System (VisualSonics) as previously described (78). Mice were lightly anesthetized with inhaled isoflurane $\left(0.2 \%\right.$ in $\left.\mathrm{O}_{2}\right)$. Left ventricle $\mathrm{M}$-mode was used, and all measurements were obtained from 3 to 6 consecutive cardiac cycles, and the average values were used for analysis. Left ventricle end-diastolic (LVDd) and end-systolic (LVDs) dimensions were measured from the M-mode traces, and fractional shortening (FS) was calculated as follows: [(LVDd - LVDs)/LVDd]. Diastolic measurements were taken at the point of maximum cavity dimension, and systolic measurements were made at the point of minimum cavity dimension, using the leading-edge method of the American Society of Echocardiography (79).

Histology and immunostaining. Deidentified human heart samples that were not suitable for transplantation and were donated for medical research were obtained through the International Institute for the Advancement of Medicine. Human and mouse hearts were fixed with 4\% paraformaldehyde overnight at $4^{\circ} \mathrm{C}$, divided into 3 parts (base, center, and apex), paraffin embedded, and cut into 5 - $\mu \mathrm{m}$ sections. For immunofluorescence analysis following deparaffinization, rehydration, and antigen retrieval, heart cross sections were incubated overnight at $4^{\circ} \mathrm{C}$ with anti-Nogo-A/B antibody (1:100, AF-6034; R\&D Systems) and anti-Nogo-A antibody (1:100, MAB3098; R\&D Systems) and then with secondary antibodies, Cy3- 
labeled donkey anti-sheep IgG (1:200, A21436; Invitrogen) and Alexa Fluor 568-conjugated donkey antimouse IgG (1:200, A10037, Invitrogen). Other myocardial sections were stained with $40 \mu \mathrm{g} / \mathrm{ml}$ wheat germ agglutinin (L4895; Sigma-Aldrich) in PBS for 1 hour at room temperature in order to label cardiomyocyte membranes. Nuclei were counterstained with DAPI. Immunofluorescence images of heart sections were captured in a Z-stack with $1-\mu \mathrm{m}$ steps using a Zeiss Axio Observer.Z1 microscope followed by deconvolution using the ZEN pro 2012 software (Carl Zeiss). To quantify the cross-sectional area as well as Nogo-positive cardiomyocytes, we used ImagePro analyzer 7.0 software (Media Cybernetics).

For immunostaining of CD45, heart cross sections were incubated overnight at $4^{\circ} \mathrm{C}$ with anti-CD45 antibody (1:50, 550539; BD Biosciences), followed by secondary goat anti-rat IgG (1:200, SC-2041; Santa Cruz Biotechnology). The staining was developed with diaminobenzene, and sections were counterstained with Mayer's hematoxylin, dehydrated, and coverslipped with mounting medium. Light microscopic images were acquired from the whole cross-sectional area of base, center, and apex using a Zeiss Axio Observer.Z1 microscope. Quantification of CD45-positive staining was performed with ImagePro analyzer 7.0 software (Media Cybernetics) and was expressed as a ratio of CD45-positive area to heart section area. At least 30 images were analyzed for each heart.

Western blotting. Western blotting was performed as previously reported (18). Protein extracts were prepared from freshly harvested heart tissue in RIPA buffer containing protease inhibitors. Western analysis was performed using $50 \mu \mathrm{g}$ heart lysates, and nitrocellulose membranes were probed with primary antiNogo-A/B antibody (1:1,000, AF-6034; R\&D Systems), anti-Nogo-A antibody (1:100, MAB3098; R\&D Systems), and anti-GAPDH antibody (1:1,000, G8795; Sigma-Aldrich). The secondary anti-mouse and anti-sheep IgGs were conjugated directly to infrared fluorescent dyes Molecular Probes Alexa Fluor 680 (Thermo Fisher Scientific) or IRDye 800 (Li-Cor) to allow direct detection on the Odyssey Infrared Imaging System (Li-Cor). The intensity of the bands was quantified with ImageJ software.

In vitro measurement of endothelial barrier function. The endothelial barrier of MLECs was assessed by measuring the resistance of a cell-covered electrode by using an Electric Cell-Substrate Impedance Sensing (ECIS) instrument (Applied BioPhysics). An 8W10E plate was incubated with $0.1 \%$ gelatin for 30 minutes. MLECs isolated from WT and Nogo-A/B-deficient mice were plated on the electrode at $4 \times 10^{4}$ cells/well. Basal transendothelial electrical resistance of a confluent MLEC monolayer was then measured.

Quantification of cardiac hypertrophy. We used the ratio of heart weight to tibia length to quantify cardiac hypertrophy. Mouse hearts were perfused with PBS $(10-15 \mathrm{ml})$ at a constant pressure of $80 \mathrm{mmHg}$ to flush out the blood, and the heart was weighed after drying the inside of the heart chambers with tissue paper.

Myriocin and SEW2871 treatment. WT and Nogo-A/B-deficient mice were i.p. injected with myriocin $(0.3 \mathrm{mg} / \mathrm{kg}, \mathrm{M} 1177$; Sigma-Aldrich) in $0.4 \%$ free fatty acid-free BSA in PBS or vehicle. Mice were treated with myriocin 24 hours before, on the same day as, and 24 hours after TAC surgery. WT mice were i.p. injected with the $\mathrm{S}_{1} \mathrm{P}_{1}$ receptor agonist SEW2871 (3 mg/kg; Cayman Chemical) or vehicle (DMSO) 1 hour before TAC surgery and every 12 hours until 72 hours after the surgery.

Cardiomyocyte isolation from adult mice. Adult murine cardiomyocytes were isolated according to an established protocol (80). Briefly, the heart was rapidly isolated, cannulated, and perfused with AfCS perfusion buffer (113 mM NaCl, $4.7 \mathrm{mM} \mathrm{KCl}, 0.6 \mathrm{mM} \mathrm{KH}_{2} \mathrm{PO}_{4}, 0.6 \mathrm{mM} \mathrm{Na}_{2} \mathrm{HPO}_{4}, 1.2 \mathrm{mM} \mathrm{MgSO}_{4}, 12 \mathrm{mM}$ $\mathrm{NaHCO}_{3}, 10 \mathrm{mM} \mathrm{KHCO}_{3}, 10 \mathrm{mM} \mathrm{HEPES}, 30 \mathrm{mM}$ taurine, $1.5 \mathrm{mM}$ glucose, and $10 \mathrm{mM}$ butanedione monoxime) for 5 minutes at a rate of $3 \mathrm{ml} / \mathrm{min}$. Hearts were then perfused with $0.65 \mathrm{mg} / \mathrm{ml}$ collagenase type 2 and $50 \mu \mathrm{M} \mathrm{CaCl}_{2}$ in AfCS perfusion buffer for 10 to 15 minutes and cut into small pieces in a protease solution $(0.65 \mathrm{mg} / \mathrm{ml}$ collagenase type $2,0.065 \mathrm{mg} / \mathrm{ml}$ protease XIV, $15 \mathrm{mg} / \mathrm{ml}$ BSA, and $50 \mu \mathrm{M}$ $\mathrm{CaCl}_{2}$ in AfCS perfusion buffer) followed by incubation at $37^{\circ} \mathrm{C}$ for 10 minutes. Gentle trituration with a pipet was used to dissociate the heart tissue until all large pieces were dispersed. After removal of enzyme solution by centrifugation at $4 \mathrm{~g}$ for 4 minutes, cells were resuspended in $15 \mathrm{mg} / \mathrm{ml} \mathrm{BSA}$ and $50 \mu \mathrm{M} \mathrm{CaCl}_{2}$ in AfCS, and cardiomyocytes were recovered on a gradient of $\mathrm{Ca}^{2+}$, with highest concentration reaching 1.8 $\mathrm{mM}$. To isolate cardiomyocytes, the cell suspension was passed through a $100-\mu \mathrm{m}$ filter and then through a $40-\mu \mathrm{m}$ filter, which retained the cardiomyocytes.

Coronary artery perfusate. The hearts of sham-operated and 3-day-banded WT and Nogo-A/B-deficient mice were rapidly excised, cannulated via the ascending aorta with a 22-gauge needle covered by a P10 tube, and perfused for 10 minutes in the Langendorff configuration under constant flow $(\sim 3 \mathrm{ml} / \mathrm{min})$ with Krebs-Henseleit solution (118.5 mM NaCl, $25.0 \mathrm{mM} \mathrm{NaHCO}_{3}, 4.7 \mathrm{mM} \mathrm{KCl}, 1.2 \mathrm{mM} \mathrm{MgSO}_{4}, 11 \mathrm{mM}$ glucose, $2 \mathrm{mM} \mathrm{CaCl}_{2}$ ) to wash out the blood. The perfusate was saturated with a mixture of $95 \% \mathrm{O}_{2}$ and $5 \%$ 
$\mathrm{CO}_{2}$ to reach $\mathrm{pH}$ 7.4. A TP650 Pump (Gaymar Industries) was used to maintain the coiled tubes containing the perfusion solutions at $37^{\circ} \mathrm{C}$. The hearts were perfused with $10 \mathrm{ml} \mathrm{Krebs-Henseleit} \mathrm{solution} \mathrm{containing}$ $0.1 \%$ fatty acid-free BSA, $10 \mathrm{mM}$ sodium glycerophosphate, $5 \mathrm{mM}$ sodium fluoride, and $1 \mathrm{mM}$ semicarbazide for 30 minutes in recirculating mode. The perfusates were then collected and sphingolipid content was analyzed by LC/MS/MS at the Lipidomic Core Facility of the Medical University of South Carolina.

Analysis of intracellular and extracellular sphingolipids in primary cultures of MLECs. MLECs were isolated from female and male WT and Nogo-A/B-deficient mice at 6 to 10 weeks of age as previously described (18). Confluent primary cultures of WT and Nogo-A/B-deficient MLECs were treated with TNF- $\alpha(50 \mu \mathrm{g} /$ $\mathrm{ml}$, no. 575202; Biolegend) or vehicle in DMEM containing 10\% charcoal-stripped FBS and Endothelial Cell Growth Supplement (no. J64516; Alfa Aesar) for 24 hours. At 19 hours after TNF- $\alpha$ treatment, the medium was replaced with DMEM containing 10\% charcoal-stripped FBS and Endothelial Cell Growth Supplement (Alfa Aesar), $10 \mathrm{mM}$ sodium glycerophosphate, $5 \mathrm{mM}$ sodium fluoride, and $1 \mathrm{mM}$ semicarbazide. After 5 hours, the medium was collected and centrifuged at $100 \mathrm{~g}$ for 10 minutes to remove dead/detached floating cells. Adherent MLECs were lysed in RIPA buffer as described above. Medium and cell lysates were analyzed for sphingolipid content by the Lipidomic Core Facility of the Medical University of South Carolina and normalized to protein concentrations. Sphingolipids in the media were expressed as $\mathrm{pg} / \mathrm{mg} / \mathrm{h}$.

Sphingolipid analysis by LC/MS/MS. Sphingolipid levels were measured in MLEC culture medium, lysates, and heart perfusates by LC/MS/MS. Levels of ceramide species, dhSph, dhSph-1-P, Sph, and S1P were analyzed by LC/MS/MS at the Lipidomics Analytical Core at the Medical University of South Carolina as previously described (81).

Preparation of murine heart microsomes. Microsomal fractions were obtained from WT and Nogo-A/Bdeficient heart tissue as previously described (18). Briefly, $1 \mathrm{ml} / \mathrm{g}$ heart of $50 \mathrm{mM}$ HEPES (pH 7.4 at $4^{\circ} \mathrm{C}$ ), $0.25 \mathrm{M}$ sucrose, and $5 \mathrm{mM}$ EDTA (adjusted to $7.4 \mathrm{pH}$ with $\mathrm{NaOH}$ ) was added to the minced hearts. Additional buffer was added to yield a $20 \%$ (w/v) suspension, and the heart was homogenized with three 15-second bursts with a Brinkman Polytron PT20. The homogenates were centrifuged for 15 minutes at $18,000 \mathrm{~g}$ at $4^{\circ} \mathrm{C}$, and the resulting supernatants were centrifuged for 1 hour at 100,000 $\mathrm{g}$. The microsomal pellets were then resuspended in $1 \mathrm{ml} / \mathrm{g}$ heart of $50 \mathrm{mM}$ HEPES $\left(\mathrm{pH} 7.4\right.$ at $\left.25^{\circ} \mathrm{C}\right), 5 \mathrm{mM}$ EDTA (pH 7.4), $5 \mathrm{mM}$ DTT, and $20 \%$ (w/v) glycerol.

Assay of microsomal SPT activity. SPT assay was performed as previously reported (18). Briefly, the assay was conducted in $0.1 \mathrm{ml}$ of $0.1 \mathrm{M}$ HEPES ( $\mathrm{pH} 8.3$ at $25^{\circ} \mathrm{C}$ ), $5 \mathrm{mM}$ DTT, $2.5 \mathrm{mM}$ EDTA (pH 7.4), $50 \mu \mathrm{M}$ pyridoxal 5'-phosphate, $0.45 \mu \mathrm{M}\left[{ }^{3} \mathrm{H}\right]$-serine, $0.2 \mathrm{mM}$ palmitoyl-CoA, and $50 \mu \mathrm{g}$ microsomes. The control contained all components except palmitoyl-CoA. The samples were incubated for 10 minutes at $37^{\circ} \mathrm{C}$, and the reaction was stopped by addition of $0.2 \mathrm{ml}$ of $0.5 \mathrm{~N} \mathrm{NH}_{4} \mathrm{OH}$, followed by $50 \mu \mathrm{NaBH}_{4}(5 \mathrm{mg} /$ $\mathrm{ml}$ ) to convert the reaction product, 3-ketosphinganine, into sphinganine. Radiolabeled lipid products were extracted using a modified Bligh and Dyer's method, as previously described (18). Briefly, $\mathrm{CHCl}_{3} / \mathrm{CH}_{3} \mathrm{OH}$ (1:2) was added to the sample, followed by $\mathrm{CHCI}_{3}$ and $0.5 \mathrm{~N} \mathrm{NH}_{4} \mathrm{OH}$. After centrifugation, the lower layer containing lipids was collected and the organic solvent was removed under a gentle stream of nitrogen gas. The samples were dissolved in $\mathrm{CHCl}_{3}$ and analyzed by thin-layer chromatography (TLC).

Assay of MLEC and HUVEC SPT activity. HUVECs and MLECs were grown to confluence in 60-mm dishes; stimulated with TNF- $\alpha(50 \mu \mathrm{g} / \mathrm{ml})$ for 8,16 , or 24 hours; and washed with PBS. Cells were scraped off the dishes in SPT reaction buffer (1 M HEPES [pH 8.3], $5 \mathrm{mM}$ DTT, $2.5 \mathrm{mM}$ EDTA [pH 7.4], and $50 \mu \mathrm{M}$ pyridoxal 5 '-phosphate) and sonicated for 15 seconds. $\left[{ }^{3} \mathrm{H}\right]$-serine $(0.45 \mu \mathrm{M})$ and 0.2 $\mathrm{mM}$ palmitoyl-CoA were added to $200 \mu \mathrm{g}$ cell lysate. The enzymatic reaction proceeded for 10 minutes at $37^{\circ} \mathrm{C}$ and was stopped by addition of $5 \mathrm{mg} / \mathrm{ml} \mathrm{NaBH}$ followed by incubation for 5 minutes at room temperature. The radiolabeled lipid products were extracted using the modified Bligh and Dyer's method as described above.

TLC analysis of lipid. Separation and quantification of lipids was performed by TLC using a silica gel 60 (Merck). Sphinganine, sphingomyelin, ceramide, and phosphatidylserine were used as standards. TLC plates were developed with chloroform, methanol, and ammonium hydroxide $(65: 25: 4)$. $\left[{ }^{3} \mathrm{H}\right]$-serinelabeled sphinganine was quantified using the RITA radioactivity TLC analyser (Raytest) and a $\left[{ }^{3} \mathrm{H}\right]$-serine calibration curve.

RNA extraction from heart tissue and real-time PCR analysis. WT and Nogo-A/B-deficient mice underwent TAC or sham surgery. Two weeks after surgery, mice were rapidly sacrificed, hearts were perfused with PBS and homogenized in liquid $\mathrm{N}_{2}$, and total RNA was extracted with Trizol (Invit- 
rogen) according to the manufacturer's protocol. Following reverse transcription using Maxima Reverse Transcriptase (Thermo Scientific), real-time PCR was performed using the iCycler Applied Biosystems 7700 and SYBR Green PCR Master Mix (Qiagen). Natriuretic peptide A and B (Nppa and $N p p b$, respectively) and $M y h 7$ primers were used to evaluate cardiac hypertrophy. Col1a1, Col3a1, and $T g f b 1$ primers were used to evaluate cardiac fibrosis. Housekeeping gene Gapdh served as internal control. Primer sequences are as follows: Nppa forward (GAGGAGAAGATGCCGGTAGA) and reverse (AGCCCTCAGTTTGCTTTT); Nppb forward (GCCAGTCTCCAGAGCAATTC) and reverse (TCCGATCCGGTCTATCTTGT); $\beta$-MHC forward (AGATGGCTGGTTTGGATGAG) and reverse (CGCACTTTCTTCTCCTGCTC); Colla1 forward (AGAGCATGACCGATGGATTC) and reverse (CCTTCTTGAGGTTGCCAGTC); Col3a1 forward (CGTAAGCACTGGTGGACAGA) and reverse (CGGCTGGAAAGAAGTCTGAG); Tgfb forward (CAACAATTCCTGGCGATACC) and reverse (GAACCCGTTGATGTCCACTT); and Gapdh forward (AGGTCGGTGTGAACGGATTTG) and reverse (TGTAGACCATGTAGTTGAGGTCA).

Measurement of myocardial edema. WT, Nogo-A/B-deficient, Nogo-A/B ${ }^{\mathrm{f} / \mathrm{f}}$, and EC-Nogo-A/B-deficient mice underwent TAC or sham surgery. In another set of experiments, WT and Nogo-A/B-deficient mice were administered i.p. injections with myriocin $(0.3 \mathrm{mg} / \mathrm{kg})$ or vehicle $(0.4 \%$ fatty acid-free BSA $)$ immediately before surgery. The day after surgery, mice were anesthetized with ketamine $(100 \mathrm{mg} / \mathrm{kg})$ and xylazine ( $5 \mathrm{mg} / \mathrm{kg}$ ) and i.v. injected with Evans Blue solution $(0.5 \% \mathrm{w} / \mathrm{v} ; 30 \mathrm{mg} / \mathrm{kg}) ; 1$ hour later the hearts were perfused from the left ventricles with PBS at a constant pressure of $80 \mathrm{mmHg}$ to gently remove the blood from the myocardial tissue. The left and right ventricles were dissected, dried overnight at $55^{\circ} \mathrm{C}$, and weighed. Albumin-bound Evans Blue dye was extracted from the myocardial tissue by addition of formamide, as previously described (82). Extravasated Evans Blue was measured spectrophotometrically at 620 nm using a standard curve of Evans Blue in formamide.

Statistics. Data are expressed as mean \pm SEM. One- or two-way ANOVA with Tukey's multiple comparison test was employed for all statistical analyses except where a 2-tailed Student's $t$ test was used. Differences were considered statistically significant at $P<0.05$. All tests were 2 -sided. GraphPad Prism software (version 6.0, GraphPad Software) was used for all statistical analyses.

Study approval. Experiments in mice were performed in agreement with the Weill Cornell Institutional Animal Care and Use Committee guidelines and with the approval of their IACUC.

\section{Author contributions}

ADL designed the experiments, interpreted the data, and wrote the manuscript. YZ designed and carried out experiments, analyzed and interpreted results, and contributed to writing of the manuscript. YH performed some of the TAC surgery. AC carried out some of the experiments. PSA performed CD45 staining, quantification, and analysis of cardiomyocyte area. MS assisted with some of the experiments. JB performed sphingolipid analysis. FJG contributed to the design of some experiments, discussion of results, and writing of the manuscript.

\section{Acknowledgments}

This work was supported by NIH grant R01HL126913-01 and a Harold S. Geneen Charitable Trust Award for Coronary Heart Disease Research to A. Di Lorenzo. We thank Timothy Hla for his critical input and support of the study.

Address correspondence to: Annarita Di Lorenzo, Center for Vascular Biology, Department of Pathology and Laboratory Medicine, Weill Cornell Medical College, 1300 York Avenue, New York, New York 10065, USA. Phone: 212.746.6476; E-mail: and2039@med.cornell.edu.

1. Hunter JJ, Chien KR. Signaling pathways for cardiac hypertrophy and failure. N Engl J Med. 1999;341(17):1276-1283.

2. Kehat I, Molkentin JD. Molecular pathways underlying cardiac remodeling during pathophysiological stimulation. Circulation. 2010;122(25):2727-2735.

3. Kai H, Kuwahara F, Tokuda K, Imaizumi T. Diastolic dysfunction in hypertensive hearts: roles of perivascular inflammation and reactive myocardial fibrosis. Hypertens Res. 2005;28(6):483-490.

4. Haudek SB, Taffet GE, Schneider MD, Mann DL. TNF provokes cardiomyocyte apoptosis and cardiac remodeling through activation of multiple cell death pathways. J Clin Invest. 2007;117(9):2692-2701.

5. McCarthy CG, Goulopoulou S, Wenceslau CF, Spitler K, Matsumoto T, Webb RC. Toll-like receptors and damage-associated 
molecular patterns: novel links between inflammation and hypertension. Am J Physiol Heart Circ Physiol. 2014;306(2):H184-H196.

6. Nicoletti A, Heudes D, Mandet C, Hinglais N, Bariety J, Michel JB. Inflammatory cells and myocardial fibrosis: spatial and temporal distribution in renovascular hypertensive rats. Cardiovasc Res. 1996;32(6):1096-1107.

7. Jiang XC, Goldberg IJ, Park TS. Sphingolipids and cardiovascular diseases: lipoprotein metabolism, atherosclerosis and cardiomyopathy. Adv Exp Med Biol. 2011;721:19-39.

8. Alewijnse AE, Peters SL. Sphingolipid signalling in the cardiovascular system: good, bad or both? Eur J Pharmacol. 2008;585(2-3):292-302.

9. Kupperman E, An S, Osborne N, Waldron S, Stainier DY. A sphingosine-1-phosphate receptor regulates cell migration during vertebrate heart development. Nature. 2000;406(6792):192-195.

10. Theilmeier G, et al. High-density lipoproteins and their constituent, sphingosine-1-phosphate, directly protect the heart against ischemia/reperfusion injury in vivo via the S1P3 lysophospholipid receptor. Circulation. 2006;114(13):1403-1409.

11. Means CK, et al. Sphingosine 1-phosphate S1P2 and S1P3 receptor-mediated Akt activation protects against in vivo myocardial ischemia-reperfusion injury. Am J Physiol Heart Circ Physiol. 2007;292(6):H2944-H2951.

12. Lee MJ, et al. Sphingosine-1-phosphate as a ligand for the G protein-coupled receptor EDG-1. Science. 1998;279(5356):15521555 .

13. Singleton PA, Dudek SM, Chiang ET, Garcia JG. Regulation of sphingosine 1-phosphate-induced endothelial cytoskeletal rearrangement and barrier enhancement by S1P1 receptor, PI3 kinase, Tiam1/Rac1, and $\alpha$-actinin. FASEB J. 2005; 19(12):16461656.

14. Rotolo JA, et al. Regulation of ceramide synthase-mediated crypt epithelium apoptosis by DNA damage repair enzymes. Cancer Res. 2010;70(3):957-967.

15. Young $\mathrm{KW}$, et al. $\mathrm{Ca}^{2+} /$ calmodulin-dependent translocation of sphingosine kinase: role in plasma membrane relocation but not activation. Cell Calcium. 2003;33(2):119-128.

16. Acevedo L, et al. A new role for Nogo as a regulator of vascular remodeling. Nat Med. 2004;10(4):382-388.

17. Di Lorenzo A, Manes TD, Davalos A, Wright PL, Sessa WC. Endothelial reticulon-4B (Nogo-B) regulates ICAM-1-mediated leukocyte transmigration and acute inflammation. Blood. 2011;117(7):2284-2295.

18. Cantalupo A, et al. Nogo-B regulates endothelial sphingolipid homeostasis to control vascular function and blood pressure. Nat Med. 2015;21(9):1028-1037.

19. Toischer K, et al. Differential cardiac remodeling in preload versus afterload. Circulation. 2010;122(10):993-1003.

20. Sarkey JP, et al. Nogo-A knockdown inhibits hypoxia/reoxygenation-induced activation of mitochondrial-dependent apoptosis in cardiomyocytes. J Mol Cell Cardiol. 2011;50(6):1044-1055.

21. Nath AK, et al. Proteomic-based detection of a protein cluster dysregulated during cardiovascular development identifies biomarkers of congenital heart defects. PLoS One. 2009;4(1):e4221.

22. Rockman HA, et al. Segregation of atrial-specific and inducible expression of an atrial natriuretic factor transgene in an in vivo murine model of cardiac hypertrophy. Proc Natl Acad Sci U S A. 1991;88(18):8277-8281.

23. Soonpaa MH, Kim KK, Pajak L, Franklin M, Field LJ. Cardiomyocyte DNA synthesis and binucleation during murine development. Am J Physiol. 1996;271(5 pt 2):H2183-H2189.

24. Porrello ER, et al. Transient regenerative potential of the neonatal mouse heart. Science. 2011;331(6020):1078-1080.

25. Sasaki R, Watanabe Y, Morishita T, Yamagata S. Estimation of the cell number of heart muscles in normal rats. Tohoku J Exp Med. 1968;95(2):177-184.

26. Beinlich CJ, Rissinger CJ, Morgan HE. Mechanisms of rapid growth in the neonatal pig heart. J Mol Cell Cardiol. $1995 ; 27(1): 273-281$.

27. Hirschy A, Schatzmann F, Ehler E, Perriard JC. Establishment of cardiac cytoarchitecture in the developing mouse heart. Dev Biol. 2006;289(2):430-441.

28. Levine B, Kalman J, Mayer L, Fillit HM, Packer M. Elevated circulating levels of tumor necrosis factor in severe chronic heart failure. N Engl J Med. 1990;323(4):236-241.

29. Feldman AM, et al. The role of tumor necrosis factor in the pathophysiology of heart failure. J Am Coll Cardiol. 2000;35(3):537544.

30. Drexler H, et al. Endothelial function in chronic congestive heart failure. Am J Cardiol. 1992;69(19):1596-1601.

31. Kubo SH, Rector TS, Bank AJ, Williams RE, Heifetz SM. Endothelium-dependent vasodilation is attenuated in patients with heart failure. Circulation. 1991;84(4):1589-1596.

32. Mann DL. Inflammatory mediators and the failing heart: past, present, and the foreseeable future. Circ Res. 2002;91(11):988998.

33. Wang Y, et al. Ephrin-B2 controls VEGF-induced angiogenesis and lymphangiogenesis. Nature. 2010;465(7297):483-486.

34. Buede R, Rinker-Schaffer C, Pinto WJ, Lester RL, Dickson RC. Cloning and characterization of LCB1, a Saccharomyces gene required for biosynthesis of the long-chain base component of sphingolipids. J Bacteriol. 1991;173(14):4325-4332.

35. Nagiec MM, Lester RL, Dickson RC. Sphingolipid synthesis: identification and characterization of mammalian cDNAs encoding the Lcb2 subunit of serine palmitoyltransferase. Gene. 1996;177(1-2):237-241.

36. Zhao C, Beeler T, Dunn T. Suppressors of the $\mathrm{Ca}^{2+}$-sensitive yeast mutant (csg2) identify genes involved in sphingolipid biosynthesis. Cloning and characterization of SCS1, a gene required for serine palmitoyltransferase activity. J Biol Chem. 1994;269(34):21480-21488.

37. Venkataraman K, et al. Vascular endothelium as a contributor of plasma sphingosine 1-phosphate. Circ Res. 2008;102(6):669676.

38. Osborne N, et al. The spinster homolog, two of hearts, is required for sphingosine 1-phosphate signaling in zebrafish. Curr Biol. 2008;18(23):1882-1888.

39. Mitra P, Oskeritzian CA, Payne SG, Beaven MA, Milstien S, Spiegel S. Role of ABCC1 in export of sphingosine-1-phosphate from mast cells. Proc Natl Acad Sci U S A. 2006;103(44):16394-16399.

40. Fukuhara S, et al. The sphingosine-1-phosphate transporter Spns2 expressed on endothelial cells regulates lymphocyte trafficking in mice. J Clin Invest. 2012;122(4):1416-1426. 
41. Slowik MR, De Luca LG, Min W, Pober JS. Ceramide is not a signal for tumor necrosis factor-induced gene expression but does cause programmed cell death in human vascular endothelial cells. Circ Res. 1996;79(4):736-747.

42. Liao R, Jain M. Isolation, culture, and functional analysis of adult mouse cardiomyocytes. Methods Mol Med. 2007;139:251-262.

43. Park TS, et al. Ceramide is a cardiotoxin in lipotoxic cardiomyopathy. J Lipid Res. 2008;49(10):2101-2112.

44. Bismuth J, Lin P, Yao Q, Chen C. Ceramide: a common pathway for atherosclerosis? Atherosclerosis. 2008;196(2):497-504.

45. Yu J, et al. Ceramide is upregulated and associated with mortality in patients with chronic heart failure. Can J Cardiol. 2015;31(3):357-363.

46. Spijkers LJ, et al. Hypertension is associated with marked alterations in sphingolipid biology: a potential role for ceramide. PLoS One. 2011;6(7):e21817.

47. Argraves KM, et al. S1P, dihydro-S1P and C24:1-ceramide levels in the HDL-containing fraction of serum inversely correlate with occurrence of ischemic heart disease. Lipids Health Dis. 2011;10:70.

48. Lee SY, et al. Cardiomyocyte specific deficiency of serine palmitoyltransferase subunit 2 reduces ceramide but leads to cardiac dysfunction. J Biol Chem. 2012;287(22):18429-18439.

49. Garcia JG, et al. Sphingosine 1-phosphate promotes endothelial cell barrier integrity by Edg-dependent cytoskeletal rearrangement. J Clin Invest. 2001;108(5):689-701.

50. Camerer E, et al. Sphingosine-1-phosphate in the plasma compartment regulates basal and inflammation-induced vascular leak in mice. J Clin Invest. 2009;119(7):1871-1879.

51. Peng X, et al. Protective effects of sphingosine 1-phosphate in murine endotoxin-induced inflammatory lung injury. Am J Respir Crit Care Med. 2004;169(11):1245-1251.

52. Adamson RH, Sarai RK, Altangerel A, Thirkill TL, Clark JF, Curry FR. Sphingosine-1-phosphate modulation of basal permeability and acute inflammatory responses in rat venular microvessels. Cardiovasc Res. 2010;88(2):344-351.

53. Okazaki M, et al. Sphingosine 1-phosphate inhibits ischemia reperfusion injury following experimental lung transplantation. Am J Transplant. 2007;7(4):751-758.

54. Lee SY, et al. Sphingosine-1-phosphate reduces hepatic ischaemia/reperfusion-induced acute kidney injury through attenuation of endothelial injury in mice. Nephrology (Carlton). 2011;16(2):163-173.

55. Oyama O, et al. The lysophospholipid mediator sphingosine-1-phosphate promotes angiogenesis in vivo in ischaemic hindlimbs of mice. Cardiovasc Res. 2008;78(2):301-307.

56. Laine GA, Allen SJ. Left ventricular myocardial edema. Lymph flow, interstitial fibrosis, and cardiac function. Circ Res. 1991;68(6):1713-1721

57. Pratt JW, et al. Acute transient coronary sinus hypertension impairs left ventricular function and induces myocardial edema. $A m$ J Physiol. 1996;271(3 pt 2):H834-H841.

58. Jones SP, et al. Myocardial ischemia-reperfusion injury is exacerbated in absence of endothelial cell nitric oxide synthase. Am $J$ Physiol. 1999;276(5 pt 2):H1567-H1573.

59. Jones SP, et al. Endothelial nitric oxide synthase overexpression attenuates myocardial reperfusion injury. Am J Physiol Heart Circ Physiol. 2004;286(1):H276-H282.

60. Jones SP, Bolli R. The ubiquitous role of nitric oxide in cardioprotection. J Mol Cell Cardiol. 2006;40(1):16-23.

61. Kubota T, et al. Dilated cardiomyopathy in transgenic mice with cardiac-specific overexpression of tumor necrosis factor-alpha. Circ Res. 1997;81(4):627-635.

62. Mann DL. Stress-activated cytokines and the heart: from adaptation to maladaptation. Annu Rev Physiol. 2003;65:81-101.

63. Sun M, et al. Tumor necrosis factor-alpha mediates cardiac remodeling and ventricular dysfunction after pressure overload state. Circulation. 2007;115(11):1398-1407.

64. Mann DL, et al. Targeted anticytokine therapy in patients with chronic heart failure: results of the Randomized Etanercept Worldwide Evaluation (RENewAL). Circulation. 2004;109(13):1594-1602.

65. Lecour S, Smith RM, Woodward B, Opie LH, Rochette L, Sack MN. Identification of a novel role for sphingolipid signaling in $\mathrm{TNF} \alpha$ and ischemic preconditioning mediated cardioprotection. J Mol Cell Cardiol. 2002;34(5):509-518.

66. Kurrelmeyer KM, et al. Endogenous tumor necrosis factor protects the adult cardiac myocyte against ischemic-induced apoptosis in a murine model of acute myocardial infarction. Proc Natl Acad Sci U S A. 2000;97(10):5456-5461.

67. Higuchi Y, McTiernan CF, Frye CB, McGowan BS, Chan TO, Feldman AM. Tumor necrosis factor receptors 1 and 2 differentially regulate survival, cardiac dysfunction, and remodeling in transgenic mice with tumor necrosis factor- $\alpha$-induced cardiomyopathy. Circulation. 2004;109(15):1892-1897.

68. Papathanasiou S, et al. Tumor necrosis factor-alpha confers cardioprotection through ectopic expression of keratins K8 and K18. Nat Med. 2015;21(9):1076-1084.

69. Sugita M, Willians M, Dulaney JT, Moser HW. Ceramidase and ceramide synthesis in human kidney and cerebellum. Description of a new alkaline ceramidase. Biochim Biophys Acta. 1975;398(1):125-131.

70. Yeboah J, et al. Endothelial dysfunction is associated with left ventricular mass (assessed using MRI) in an adult population (MESA). J Hum Hypertens. 2011;25(1):25-31.

71. Perticone F, Maio R, Ceravolo R, Cosco C, Cloro C, Mattioli PL. Relationship between left ventricular mass and endothelium-dependent vasodilation in never-treated hypertensive patients. Circulation. 1999;99(15):1991-1996.

72. Millgård J, et al. Left ventricular hypertrophy is associated with an attenuated endothelium-dependent vasodilation in hypertensive men. Blood Press. 2000;9(6):309-314.

73. Hamasaki S, Al Suwaidi J, Higano ST, Miyauchi K, Holmes DR Jr, Lerman A. Attenuated coronary flow reserve and vascular remodeling in patients with hypertension and left ventricular hypertrophy. J Am Coll Cardiol. 2000;35(6):1654-1660.

74. Scherrer-Crosbie M, et al. Endothelial nitric oxide synthase limits left ventricular remodeling after myocardial infarction in mice. Circulation. 2001;104(11):1286-1291.

75. Moens AL, et al. Reversal of cardiac hypertrophy and fibrosis from pressure overload by tetrahydrobiopterin: efficacy of recoupling nitric oxide synthase as a therapeutic strategy. Circulation. 2008;117(20):2626-2636.

76. Landmesser U, et al. Statin-induced improvement of endothelial progenitor cell mobilization, myocardial neovascularization, left ventricular function, and survival after experimental myocardial infarction requires endothelial nitric oxide synthase. Circula- 
tion. 2004;110(14):1933-1939.

77. Zheng B, Ho C, Li S, Keirstead H, Steward O, Tessier-Lavigne M. Lack of enhanced spinal regeneration in Nogo-deficient mice. Neuron. 2003;38(2):213-224

78. Krieger MH, Di Lorenzo A, Teutsch C, Kauser K, Sessa WC. Telmisartan regresses left ventricular hypertrophy in caveolin-1-deficient mice. Lab Invest. 2010;90(11):1573-1581.

79. Lang RM, et al. Recommendations for chamber quantification: a report from the American Society of Echocardiography's Guidelines and Standards Committee and the Chamber Quantification Writing Group, developed in conjunction with the European Association of Echocardiography, a branch of the European Society of Cardiology. J Am Soc Echocardiogr. 2005;18(12):1440-1463.

80. Huang Y, et al. Cardiac myocyte-specific HIF-1 $\alpha$ deletion alters vascularization, energy availability, calcium flux, and contractility in the normoxic heart. FASEB J. 2004;18(10):1138-1140.

81. Bielawski J, Pierce JS, Snider J, Rembiesa B, Szulc ZM, Bielawska A. Sphingolipid analysis by high performance liquid chromatography-tandem mass spectrometry (HPLC-MS/MS). Adv Exp Med Biol. 2010;688:46-59.

82. Di Lorenzo A, et al. eNOS-derived nitric oxide regulates endothelial barrier function through VE-cadherin and Rho GTPases. J Cell Sci. 2013;126(pt 24):5541-5552. 